\title{
DEVELOPMENT OF A QUANTITATIVE HIGH THROUGHPUT METHOD FOR THE EARLY DETECTION OF CORROSION ON COATED ALUMINUM
}

\author{
A Thesis \\ presented to \\ the Faculty of California Polytechnic State University, \\ San Luis Obispo
}

\author{
In Partial Fulfillment \\ of the Requirements for the Degree \\ Master of Science in Polymers and Coatings \\ by \\ Jeffrey Clayton Foster
}

June 2012 
(C) 2012

Jeffrey Clayton Foster ALL RIGHTS RESERVED 


\section{COMMITTEE MEMBERSHIP}

TITLE:

AUTHOR:

DATE SUBMITTED:

COMMITTEE CHAIR:

COMMITTEE MEMBER:

COMMITTEE MEMBER:
Development of a Quantitative High Throughput Method for the Early Detection of Corrosion on Coated Aluminum

Jeffrey Clayton Foster

June 2012
Chad Immoos, Associate Professor

Raymond Fernando, Professor

Erik Sapper, Advanced Research Teams, Chemical Technology, Boeing Research \& Technology 


\begin{abstract}
Development of a Quantitative High Throughput Method for the Early Detection of Corrosion on Coated Aluminum

Jeffrey Clayton Foster
\end{abstract}

Eight fluorescent indicators were evaluated for their ability to detect the corrosion of aluminum metal substrates. The fluorophore Rhodamine-salicylaldehyde (RSA) was selected as a candidate for further study based on its ability to bind and detect aluminum ions at low concentrations, its selectivity for aluminum ions, its long-term stability, its solubility in our solvent-based epoxy formulation, and its compatibility with our testing method.

A recent publication suggested that an alternative method of fluorescence activation was possible - an acid-promoted ring opening that occurred in the absence of metal ions. To prove the capability of RSA to bind aluminum ions, thorough ${ }^{1} \mathrm{H}$ and ${ }^{27} \mathrm{Al}$ nuclear magnetic resonance spectroscopy, mass spectrometry, and fluorescence analysis was conducted. It was found that RSA bound aluminum ions, with a preferred binding stoichiometry of 2:1 RSA/Al.

Long-term immersion and salt spray corrosion studies were conducted to investigate the ability of RSA to detect corrosion on aluminum substrates. Aluminum panels were coated with epoxy coatings that contained the fluorescent indicator. Following optimization, a linear relationship between corroded area and time of immersion/exposure to salt fog was observed.

Keywords: Corrosion of aluminum, fluorophore, fluorescent indicator. 


\section{ACKNOWLEDGMENTS}

I would like to thank the members of my graduate committee-Dr. Raymond Fernando and Erik Sapper — and especially my advisor, Dr. Chad Immoos, for their guidance and support, and for ultimately allowing me to complete this masterwork of applied science.

I also wish to acknowledge The Boeing Company for sponsoring this work.

Without their support, my thesis research would not have been possible.

I would like to thank the University of California Santa Barbra for assistance with

mass spectrometry measurements and Dr. Seth Bush for help and guidance when

conducting aluminum nuclear magnetic resonance spectroscopy.

Finally, I wish to thank and acknowledge a number of people who have contributed advice, expertise, and assistance to this work, namely Dr. Philip Costanzo, James Lukens, Matt Larsen, Doug Amato, and Tyler Bell (for scratching my back). 


\section{TABLE OF CONTENTS}

LIST OF ABBREVIATIONS............................................................................................. vi

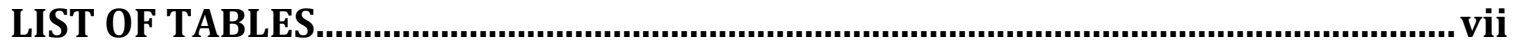

LIST OF FIGURES.......................................................................................................

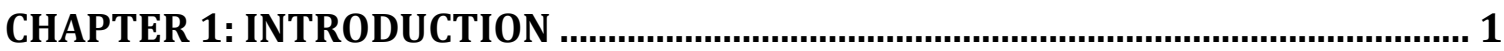

1.1 Corrosion's Effect on the Manufacturing Industry …................................................. 1

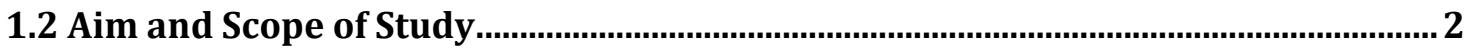

Chapter 2: Background of Study …...................................................................... 5

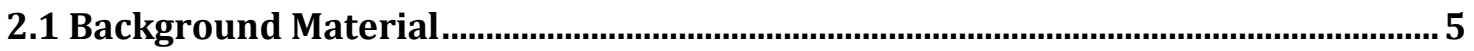

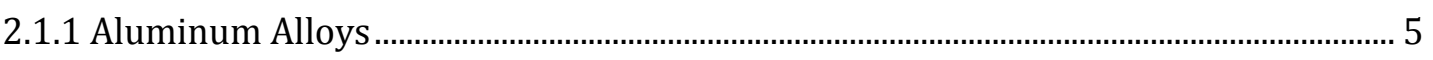

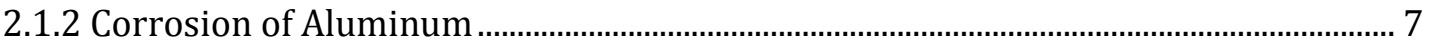

2.1.2.1 Intergranular Corrosion and Exfoliation ${ }^{2}$............................................................................... 8

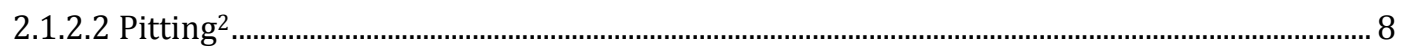

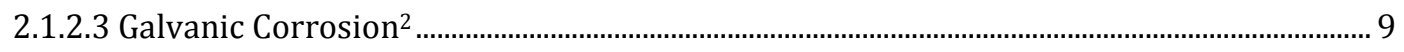

2.1.3 Inhibition and Passivation of Corrosion .........................................................................

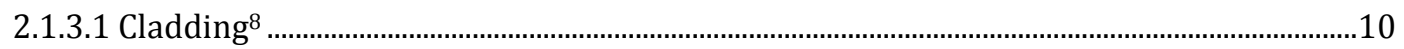

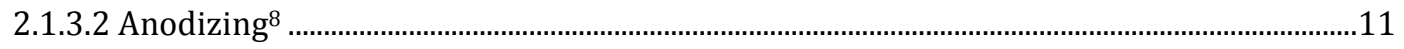

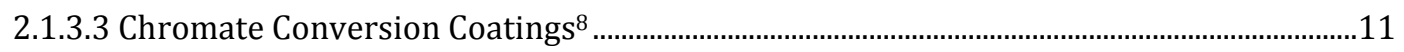

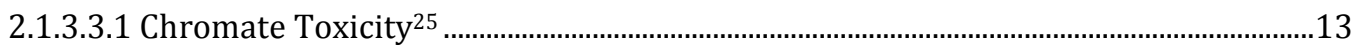

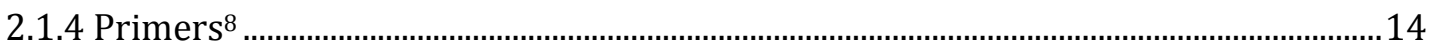

2.1.4.1 Primer Oxygen and Water Permeability 8 ..............................................................................15

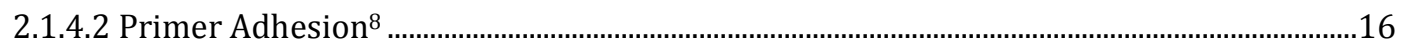

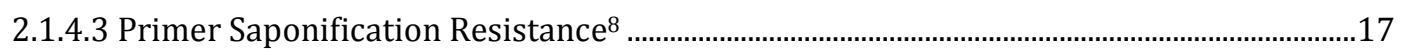

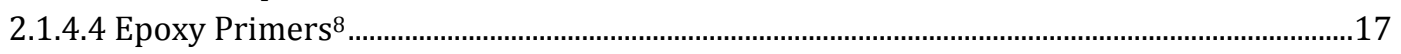

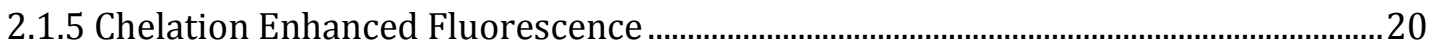

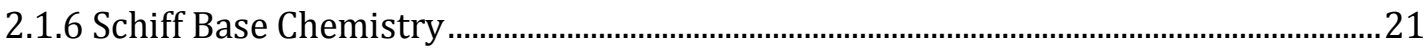

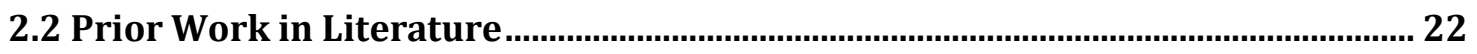

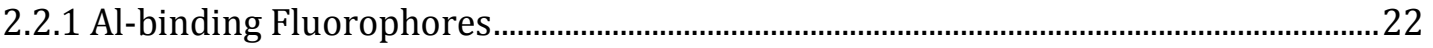

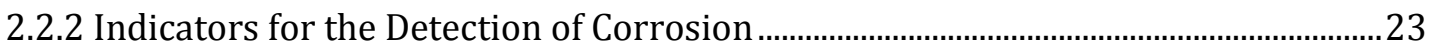

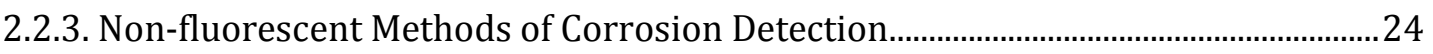

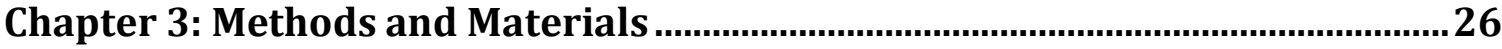

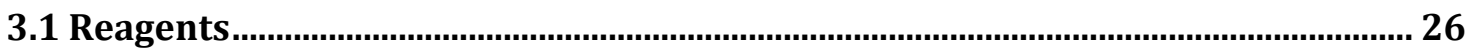

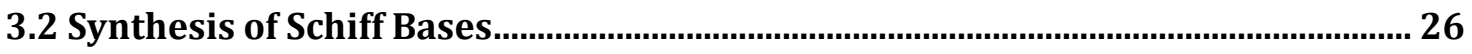

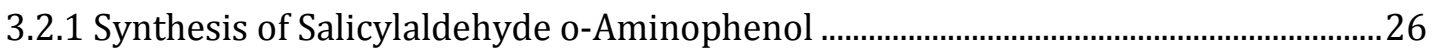

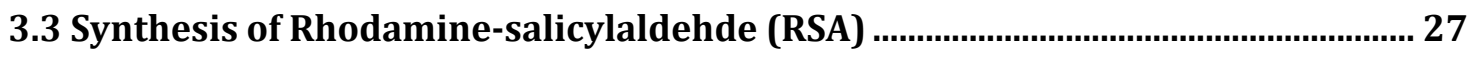

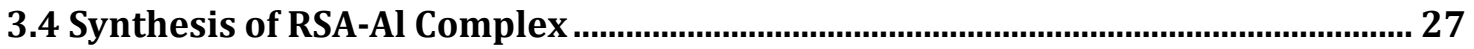

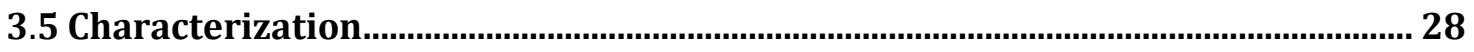

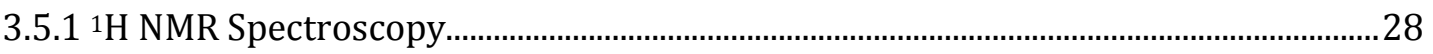

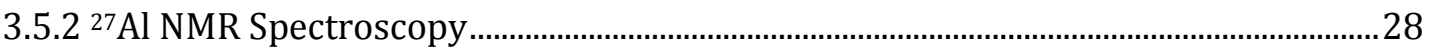

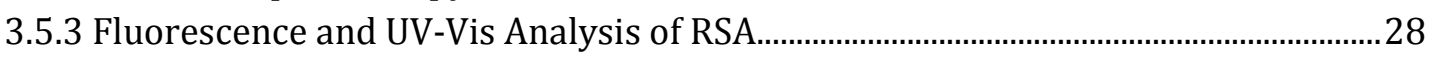

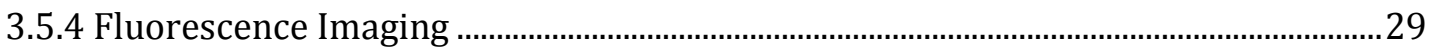




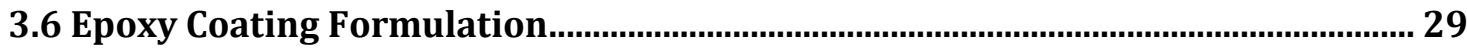

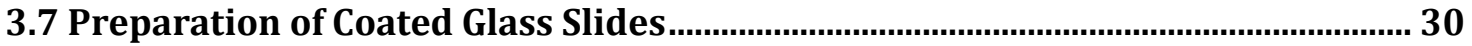

3.8 Preparation of Coated Aluminum Panels................................................................ 30

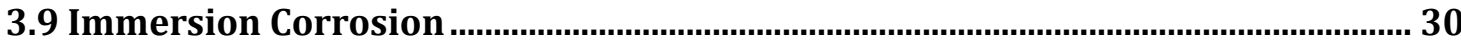

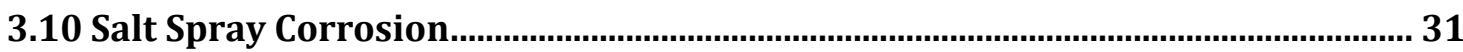

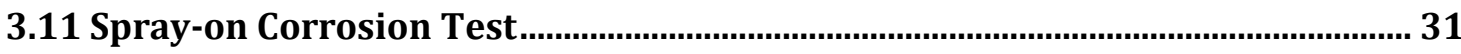

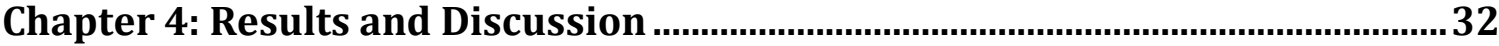

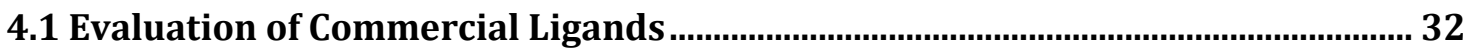

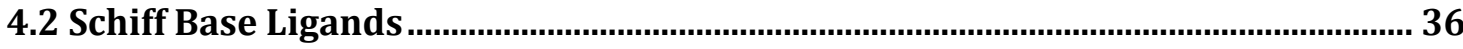

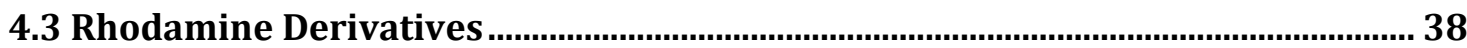

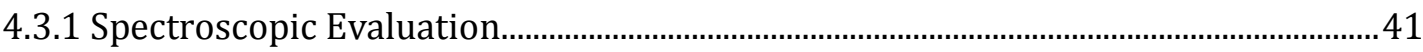

4.3.2 Binding of Aluminum Ions by Rhodamine-based Fluorophores ................................... 44

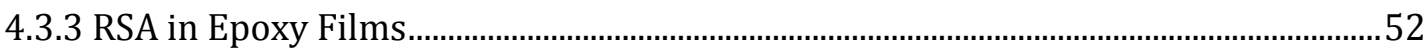

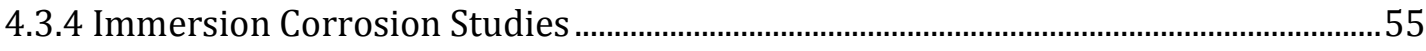

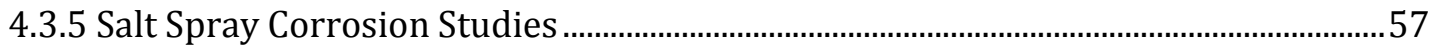

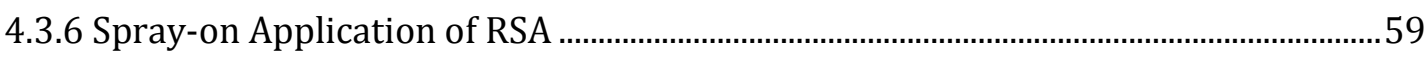

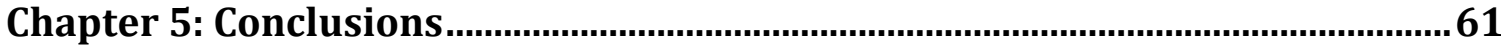

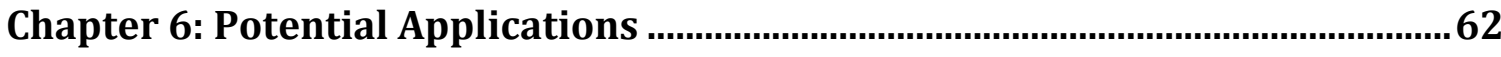

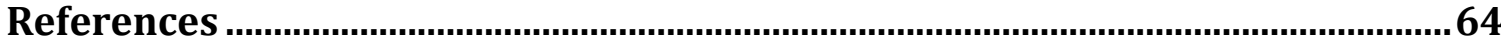

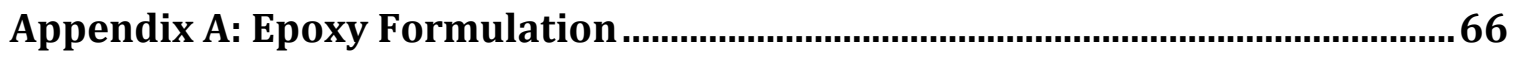




\section{LIST OF ABBREVIATIONS}

GP - Guinier-Preston

DNA - Deoxyribonucleic acid

2K - Two component

UV - Ultra Violet

CHEF - Chelation-enhanced fluorescence

CHEQ - Chelation-enhanced quenching

ACS - Americal Chemical Society

8-HQS - 8-Hydroxyquinoline-5-sulfonic acid monohydrate

SOAP - Salicylaldehyde o-aminophenol

RAc - Rhodamine-acetone

RSA - Rhodamine-salicylaldehyde

NMR - Nuclear magnetic resonance

HCl - Hydrochloric acid

DCI - Deuterium chloride 


\section{LIST OF TABLES}

page

Table 1. Composition of aluminum alloy 2024. ${ }^{2} \quad 6$

Table 2. Galvanic series in flowing sea water. ${ }^{7} \quad 10$

Table 3. Solubilities of commercial ligands in selected solvents. 33

Table 4. Excitation wavelengths found for commercial indicators. 34

Table 5. Summary of coated glass slide immersion tests. 35 


\section{LIST OF FIGURES}

Figure 1. Fluorophores evaluated as potential indicators of aluminum corrosion

page

Figure 2. Generalized diagram of CHEF.

Figure 3. Fluorescence activation of Lumogallion following the addition of aluminum nitrate.

Figure 4. Commercial ligands evaluated as potential corrosion indicators.

Figure 5. Optical microscope image of Morin (left) and 8-HQS (right) showing poorly dispersed dye aggregates and epoxy coating imperfections - the result of the solvents utilized and poor solubility in the dry film.

Figure 6. Schiff bases evaluated as potential indicators of corrosion.

Figure 7. Fluorescence response of a $50 \mu \mathrm{M}$ solution of Compound 5 in ethanol with increasing amounts of aluminum nitrate.

Figure 8. Loss of fluorescence intensity of a $50 \mu \mathrm{M}$ solution Compound 5 with an equimolar amount of aluminum nitrate in ethanol after 48 hours in solution-the result of imine bond hydrolysis.

Figure 9. Rhodamine ligands evaluated for their efficacy as indicators of the corrosion of aluminum substrates.

Figure 10. Fluorescence response of RAc in the presence of various metal ions.

Figure 11. Similarity of the metal binding sites of RSA and SOAP.

Figure 12. Fluorescence response of RSA in the presence of various metal ions.

Figure 13. Side by side comparison of titration of a solution of RSA in acetonitrile with $\mathrm{HCl}$ (left) and aluminum nitrate (right).

Figure 14. Plots of emission intensity as a function of equivalents of titrant for $\mathrm{HCl}$ and aluminum nitrate fluorescence titration experiments.

Figure 15. ${ }^{1} \mathrm{H}$ NMR spectrum of RSA in acetonitrile-d3.

Figure 16. ${ }^{1} \mathrm{H}$ NMR evaluation of RSA and its starting materials. Spectra are, from bottom to top: salicylaldehyde, rhodamine-salicylaldehyde (RSA), RSA and Al, RSA and DCl. All experiments were conducted in acetonitrile-d3.

Figure 17. Spectra are, from bottom to top: aluminum nitrate in deuterium oxide (external standard), $\mathrm{Al}^{3+}$ in 50:50 toluene/methanol-d4; a 3:2 excess of aluminum ions to RSA in the same solvent; a stoichiometric amount of RSA; a 3:2 excess of RSA.

Figure 18. Mass spectrum of the RSA-Al complex.

Figure 19. Job plot of RSA with $\mathrm{Al}^{3+}$ in acetonitrile; Y-intercept $=0.352$

Figure 20. Imaging with the Typhoon Trio+ revealed an increase in fluorescence emission 
intensity after soaking for one hour in aluminum nitrate solution.

Figure 21. Aggregation of the RSA-Al complex (top) and the improvement in dispersion following the addition of an anionic surfactant.

Figure 22. Calibration of the RSA-Al complex in epoxy coatings. Shown are the

fluorescence image (left) and the accompanying calibration plot (right).

Figure 23. Fluorescence images of aluminum coupons after submerging in $\mathrm{NaCl}$ solution for a period of time.

Figure 24. Plot of corroded area as a function of time. Data points that differ more than $30 \%$ from the linear trend are colored blue.

Figure 25. Fluorescence images of aluminum panels after salt fog exposure.

Figure 26. Plot of average emission intensity over time.

Figure 27. Fluorescence images of corroded aluminum panels sprayed with a $1 \% \mathrm{w} / \mathrm{v}$ solution of RSA. 


\section{CHAPTER 1: INTRODUCTION}

The corrosion of materials represents a major hazard to the longevity of infrastructure, vehicles, and other metal-based products, and more generally to any metallic object exposed to the damaging effects of the environment. As we will see, the prevention of corrosion is a multi-billion dollar industry that begins with the processing of raw materials and follows the manufacturing process to the end product. A number of methods exist to combat this natural, yet destructive, phenomenon including corrosion-resistant coatings, corrosion inhibitors, and passivation. Despite the practice of corrosion inhibition, corrosion inevitably occurs. Therefore, the ability to detect, and further to evaluate, the existence and extent of corrosion at each stage of the lifecycle of a material is paramount.

\subsection{Corrosion's Effect on the Manufacturing Industry}

Corrosion causes damage to industrial products ranging from automobiles to pipelines, home appliances to infrastructure such as bridges and power lines, and drinking water systems to public buildings. According to a recent national corrosion study, the direct annual cost of metallic corrosion is $\$ 276$ billion, a value that makes

up $3.1 \%$ of the U.S. gross domestic product. ${ }^{1}$ Per capita, the annual cost of corrosion for U.S. residents is approximately $\$ 970 .{ }^{1}$ In comparison, the normalized losses from weather-related disasters are estimated at $\$ 380$ billion over the past 22 years (only $\$ 17$ billion annually). ${ }^{1}$ The incurred cost of corrosion stems not only from the lost material, but also from indirect costs such as outages, delays, failures, and litigation, taxes and overhead on the cost of corrosion inhibition, and maintenance fees. 
It has been estimated that, through the use of "optimum corrosion management practices", 25 to $30 \%$ of annual corrosion costs could be saved. ${ }^{1}$ Included in the list of preventative strategies provided by the National Association of Corrosion Engineers is the development of advanced life-prediction and performance assessment methods.

\subsection{Aim and Scope of Study}

The most common method for detecting corrosion, and further for evaluating the efficacy of corrosion inhibitors, is a visual inspection of the coated substrates for pitting, blistering, and other signs of corrosion at various time intervals under corrosive conditions. This method is time-consuming, labor intensive, subjective, and non-quantitative, providing little data with which to compare the effectiveness of various corrosion inhibitor systems. To facilitate these studies, we report a quantitative method to evaluate the extent of corrosion on a coated aluminum alloy surface utilizing $\mathrm{Al}^{3+}$ responsive fluorescent indicators. The scope of this study encompassed the following objectives:

1. The selection of an appropriate $\mathrm{Al}^{3+}$-responsive fluorophore for use as a corrosion indicator on epoxy coated $\mathrm{Al}-2024 \mathrm{~T} 3$ by evaluating commercially available fluorophores and synthesizing potential fluorophore candidates.

2. The development of a rapid and quantitative method for evaluating the extent of corrosion of an epoxy/polyamide-coated aluminum substrate.

3. The collection of preliminary results on the performance of this method in the evaluation of chromate and non-chromate inhibitors of corrosion. 
The process of evaluating corrosion indicators involved the determination of solubility, sensitivity for aluminum, and compatibility with our instrumentation, namely the Typhoon Trio+ confocal imager. The following indicators were evaluated for their ability to detect the corrosion of aluminum alloys.<smiles>O=c1c(O)c(-c2ccc(O)cc2O)oc2cc(O)cc(O)c12</smiles>

1<smiles>CCN(CC)c1ccc(/C=N/c2c[nH]c(=O)[nH]c2=O)c(O)c1</smiles>

4<smiles></smiles><smiles>O=S(=O)(O)c1ccc(O)c2ncccc12</smiles>

2

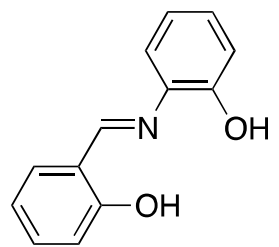

5<smiles>O=S(=O)(O)c1cc(Cl)cc(N=Nc2ccc(O)cc2O)c1O</smiles>

3

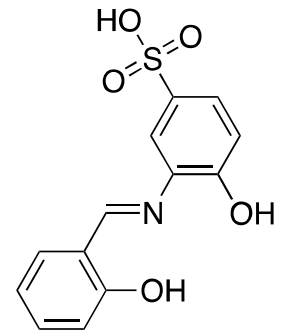

6

Figure 1. Fluorophores evaluated as potential indicators of aluminum corrosion. Once an appropriate fluorophore had been selected, studies on glass slides were conducted to assess the feasibility of our method. Following this, corrosion studies on aluminum panels were conducted, from which a quantitative relationship was observed between the fluorescent area and the amount of time under corrosive conditions. The fluorescent area corresponds directly to the quantity of indicator 
molecules activated by chelation and by extension the amount of aluminum ions produced during the process of corrosion. 


\section{Chapter 2: Background of Study}

\subsection{Background Material}

To design fluorescent indicators for the detection of corrosion, the metallurgy of aluminum alloys, their susceptibility to corrosion, and the common methods of corrosion prevention must be understood.

\subsubsection{Aluminum Alloys}

Metal alloys are prevalent in manufacturing industries due to their enhanced mechanical strength. An alloy is a metallic solid solution composed of two or more elements - a mixture of a solid-state solvent and solute that does not affect the crystal structure of the solvent and results in a single, homogeneous phase. ${ }^{2}$ While the physical properties of an alloy, for example, its density, reactivity, Young's modulus, and thermal and electrical conductivities, might not differ from those of its constituent elements, often the engineering properties such as tensile and shear strength are substantially different. These improved mechanical properties derive in large part from the difference in size of the alloy's component atoms. Larger atoms exert a compressive force on their neighbors, and small atoms a tensile force. ${ }^{3}$ The combination of these factors helps the alloy resist deformation.

Aluminum alloy 2024-T3 is a high strength, low-density alloy widely used in the aerospace industry due to its superior mechanical properties. ${ }^{2}$ The material is agehardened, gaining its excellent tensile strength, reported to be as high as 70,000 psi, damage tolerance, and fatigue resistance from a combination of alloy additions, heattreatments, and aging. ${ }^{2,4,5}$ The first digit of the alloy's designation indicates the major alloying element, in this case copper. ${ }^{2}$ The last two digits identify the specific alloy, 
with 2024 being used for the fuselage skin and frame along with other structural components. ${ }^{2}$ The final component, T3, specifies that the alloy has been solution-heat treated and then cold worked. ${ }^{2}$

Al 2024-T3 contains appreciable quantities of $\mathrm{Cu}, \mathrm{Mg}$, and $\mathrm{Mn}$ and trace amounts of $\mathrm{Si}, \mathrm{Fe}, \mathrm{Zn}, \mathrm{Cr}$, and Ti. The composition of this alloy, given in terms of weight percent, is shown in Table $1 .^{2,4}$

Table 1. Composition of aluminum alloy $2024 .^{2}$

\begin{tabular}{|c|c|c|c|c|c|}
\hline \multicolumn{7}{|c|}{ Elemental \% } \\
\hline $\mathbf{A l}$ & $\mathbf{C u}$ & $\mathbf{M n}$ & $\mathbf{M g}$ & $\mathbf{Z n}$ & $\mathbf{C r}$ \\
\hline $90+$ & 4.40 & 0.6 & 1.5 & trace & trace \\
\hline
\end{tabular}

These added strengtheners have been found to have maximum solubilities at elevated temperatures. ${ }^{4}$ Therefore, the alloy is heat treated to maximize the mechanical properties of the final product. The heat treatment schedule follows a typical sequence: a solution heat treatment in a single phase region, a rapid quenching step, cold working, and finally solid solution decomposition through aging. ${ }^{4}$ During the initial heating step, the alloying metals form an equilibrium solid solution with Al. ${ }^{4,5}$ Quenching of the solid solution results in supersaturation and the precipitation of insoluble intermetallics. ${ }^{5}$ Those quantities of $\mathrm{Cu}$ and $\mathrm{Mg}$ that remain in solution form a dispersion of particulate clusters. Guinier-Preston (GP) zones—aggregations of atoms that are composed of high concentrations of solute metals-form as these clusters grow. ${ }^{5}$ Cold working increases dislocation density, facilitating the creation of semi-coherent and incoherent phases around lattice irregularities such as GP zones. ${ }^{4}$ These out-of-phase hardening particles are typically $\mathrm{Al}_{2} \mathrm{Cu}$ and $\mathrm{Al}_{2} \mathrm{CuMg}$ amalgams, are largely responsible for the enhanced mechanical properties, and can exist at 
diameters up to a micrometer. ${ }^{5,6}$ The optimal hardness is obtained by reaching a critical dispersion of these hardening particles, thereby maximizing the strain on the material around precipitates and GP zones in the alloy and in turn maximizing resistance to dislocation motion. ${ }^{4}$

However, the increased strength properties come at a cost. The heterogeneous microstructure of the alloy makes the material inherently susceptible to localized corrosion. ${ }^{2}$ Compositional differences between the matrix and intermetallic particles lead to the formation of galvanic couples at the interfaces of the heterogeneous microstructure. $^{2}$ Anodic and cathodic reactions occur, causing the more active metal to become an anode and preferentially dissolve away. ${ }^{2,4}$ For Al-2024 T3 it has been found that the copper-containing intermetallic regions are typically noble to the surrounding aluminum matrix. During exposure to an electrolyte such as an aqueous solution of $\mathrm{NaCl}$, these $\mathrm{Cu}$-containing precipitates support rapid cathodic kinetics. ${ }^{5}$ Therefore, cathodic intermetallic particles drive corrosion in the surrounding material. The introduction of alloying metals results in enhanced mechanical properties, but these gains are coupled with the cost of increased corrosion susceptibility.

\subsubsection{Corrosion of Aluminum}

Corrosion is a natural process, occurring as the result of chemical reactions between the material, typically a metal, and the environment. The quintessential example of this phenomenon is the rusting of iron —-the oxidation of iron arising from a reaction between the metal surface and an oxidizing agent such as $\mathrm{O}_{2}$ to produce iron oxide. Copper containing alloys, such as Al-2024 T3, are susceptible to various forms of 
corrosion. A brief discussion of the relevant corrosion mechanisms is presented below.

\subsubsection{Intergranular Corrosion and Exfoliation ${ }^{2}$}

Intergranuluar corrosion occurs at the boundaries between micro-scale granules and the matrix metal. At such interfaces, the aluminum matrix is anodic to the granule, resulting in the corrosion of the aluminum metal in the presence of an electrolyte. The corrosion follows a lamellar pattern along the granular boundary and could manifest as exfoliation corrosion at the macroscopic scale if severe. Exfoliation produces layers of aluminum that protrude from the metal surface. The heating schedule and the concentrations of the alloying metals can be tuned to reduce susceptibility to this type of corrosion, as these parameters determine the size and quantity of dissolved intergranular material. However, it is important to reiterate that modifications to the alloy itself, in an attempt to minimize its vulnerability to corrosion, often come at the cost of reduced strength.

\subsubsection{Pitting ${ }^{2}$}

Should the area of the cathodic area be large relative to the anodic area, pitting corrosion can occur. This extensively localized form of corrosion results in the formation of holes or pits in the metal that can eventually lead to exfoliation if left unattended. The mechanism of this corrosion process is typically initiated by galvanic corrosion and sustained as chloride anions migrate to the pit and increase the local

acidity. Aluminum corrodes anodically at the pit site, becoming oxidized to $\mathrm{Al}^{3+}$ according to the equation:

$$
A l \rightarrow A l^{3+}+3 e^{-}
$$


Electrons produced by this reaction flow to the cathode and are discharged in the cathodic reaction:

$$
\frac{1}{2} \mathrm{O}_{2}+\mathrm{H}_{2} \mathrm{O}+2 e^{-} \rightarrow 2 \mathrm{OH}^{-}
$$

The newly formed aluminum cations draw more chloride anions to the pit, further decreasing the $\mathrm{pH}$ of the electrolyte:

$$
\mathrm{AlCl}_{3}+3 \mathrm{H}_{2} \mathrm{O} \rightarrow \mathrm{Al}(\mathrm{OH})_{3}+3 \mathrm{HCl}
$$

As the $\mathrm{pH}$ continues to decrease, the corrosion process becomes increasingly accelerated via the autocatalytic mechanism described above.

\subsubsection{Galvanic Corrosion ${ }^{2}$}

When two metals have physical contact with each other and are submerged in an electrolyte solution an electrochemical cell, known as a Galvanic couple, is formed in which the anode corrodes at an accelerated rate, and the rate of corrosion of the cathode is retarded. The metal determines the electrode type. Noble metals, so named for their resistance to corrosion and oxidation, are less reactive and serve as the cathode. The anode is an active metal, i.e. one having a low oxidation half-reaction potential. Taken together, both noble and active metals form a hierarchy known as a galvanic series. Using this information, the relative corrosion rates of elements within metal alloys can be predicted. The galvanic series is summarized in Table 2 . 
Table 2. Galvanic series in flowing sea water. ${ }^{7}$

\begin{tabular}{|l|c|}
\hline \multicolumn{1}{|c|}{ Material } & Electrode Potential (V)* \\
\hline Graphite & +0.25 \\
\hline Platinum & +0.15 \\
\hline Titanium & -0.1 \\
\hline Silver & -0.31 \\
\hline Copper & -0.36 \\
\hline Cast Iron & -0.61 \\
\hline Aluminum & $\mathbf{- 0 . 7 9}$ \\
\hline Zinc & -1.03 \\
\hline
\end{tabular}

*Saturated calomel half-cell.

The galvanic properties of metal alloys are often exploited to produce corrosionresistant materials. For example, zinc is frequently employed as a sacrificial metal, preferentially corroding before the metal it is alloyed with.

\subsubsection{Inhibition and Passivation of Corrosion}

Several methods exist to decrease the rate of corrosion. The class of materials that decrease the rate of corrosion are called corrosion inhibitors, an important subclass of which are those that retard the anodic reaction, known as passivators. Common corrosion inhibition mechanisms are cladding, anodizing, chromate conversion coatings, and primers.

\subsubsection{Cladding ${ }^{8}$}

Cladding refers to the process by which alloys are "clad", or coated, with a thin layer of pure metal. The coating is typically applied by submerging an alloy sheet into molten aluminum, creating a surface barrier that resists corrosion. The cladding is anodic to the alloy, protecting the underlying metal from electrochemical corrosion should it be penetrated. 


\subsubsection{Anodizing ${ }^{8}$}

The process of anodization involves the conversion of aluminum on the surface of the alloy to aluminum oxide. Aluminum acts as an anode, suspended in an aqueous electrolyte through which a current is passed. Oxygen is produced at the anodic surface via the hydrolysis of water, resulting in the formation of aluminum oxide. The overall reaction occurring during anodization is:

$$
2 \mathrm{Al}+3 \mathrm{H}_{2} \mathrm{O} \rightarrow \mathrm{Al}_{2} \mathrm{O}_{3}+3 \mathrm{H}_{2}
$$

Following anodization, the metal surface is littered with millions of micronsized pores lined with amorphous aluminum oxide. A subsequent process, known as sealing, is required to convert the amorphous oxides to monohydrates that fully or partially close the pores depending on parameters of the procedure. If the material is to be painted (coated), the pores may not be completely sealed to improve adhesion at the substrate/film interface.

In comparison, anodization provides better corrosion protection than chromate conversion coatings (see below). However, anodization is less common in the aerospace industry, owing to the fact that the process is neither cost efficient nor suitable for large or awkwardly-shaped parts.

\subsubsection{Chromate Conversion Coatings ${ }^{8}$}

Chromates have been utilized as pigments and conversion coatings to inhibit the corrosion of alloys due to their ability to retard anodic and cathodic kinetics and to self-heal should pitting or trenching be initiated on the metal surface. Chromate conversion coatings are applied under acidic conditions to ensure $\mathrm{Cr}_{2} \mathrm{O}_{7}{ }^{2-}$ is the predominate chromate species in solution. The coating conversion proceeds via a 
two-step mechanism. In the first step, electrochemical activity on the metal surface results in the reduction of the $\mathrm{Cr}^{6+}$ species in solution to an insoluble $\mathrm{Cr}^{3+}$ species, accompanied by oxidation of the surrounding matrix material. A barrier, typically 0.01 to $3 \mu \mathrm{m}$ thick, is formed by this process, consisting of $\mathrm{Al}_{2} \mathrm{O}_{3}$ and $\mathrm{Cr}(\mathrm{OH})_{3}$, that protects against corrosion. The monolayer formed in this initial step substantially reduces electron transfer and oxidation on the surface. Following deposition, the octahedral $\mathrm{Cr}(\mathrm{OH})_{3}$ units polymerize, resulting in enhanced inhibition of oxygen and $\mathrm{Cr}^{6+}$ reduction.

After the formation of the $\mathrm{Cr}^{3+}$ film, the second stage of chromate conversion occurs when $\mathrm{Cr}^{6+}$ ions bind to the surface of the monolayer. A reversible $\mathrm{Cr}^{3+}-\mathrm{O}-\mathrm{Cr}^{6+}$ bond is formed as a result, increasing the coating thickness and imparting additional corrosion resistance. Moreover, the presence of $\mathrm{Cr}^{6+}$ that is reversibly bound to the oxide film provides an additional benefit. In essence, the coating is imparted with a reservoir from which $\mathrm{Cr}^{6+}$ can be released according to the equilibrium:

$$
\mathrm{Cr}^{3+} \mathrm{OH}^{-}(s)+\mathrm{Cr}^{6+} \mathrm{O}_{4}^{2-}(a q)+4 \mathrm{H}^{+}(a q) \leftrightarrow \mathrm{Cr}^{3+}-\mathrm{O}-\mathrm{Cr}^{6+} \mathrm{O}_{3}^{-}(s)+4 \mathrm{H}_{2} \mathrm{O}
$$

The equilibrium indicates that $\mathrm{Cr}^{6+}$ release is $\mathrm{pH}$ dependent, with alkalinity favoring the release of the ion into the surrounding solution. The release of $\mathrm{Cr}^{6+}$ allows for the chromate conversion coating to heal minor defects in the metal surface, as this ion can participate in the electrochemical reactions described above. For example, increased alkalinity as the result of oxygen reduction near a cathodic site would trigger the release of $\mathrm{Cr}^{6+}$, which could then migrate to the defect and be reduced to $\mathrm{Cr}^{3+}$ to reform the protective oxide barrier. 
Chromate conversion coatings have an additional, indirect advantage. The prevalence of polar functionality introduced by the chromate conversion process leads to increased surface energy, which improves the adhesion of paints applied on top of the metal oxide layer.

While chromate conversion coatings are effective in inhibiting corrosion, they suffer from several significant disadvantages:

(1) Hexavalent chromium compounds are toxic, and are considered hazardous waste, necessitating the observation of proper collection and disposal.

(2) The adhesion of primers to the chromate-converted surface is often so tenacious that their removal is difficult, requiring hours of labor and vast quantities of chemical stripper to remove.

The chromate film is brittle, especially at low temperatures. This can result in cracking, chipping, and adhesion failure at joints and seams, and around rivets and fasteners.

Chromate is also used as a pigment, incorporated into primers and coatings. The mechanism of action is similar to chromate conversion coatings, with the release of hexavalent chromium leading to preferential reduction of chromium during galvanic corrosion reactions.

\subsection{Chromate Toxicity ${ }^{25}$}

Hexavalent chromate is a known carcinogen. $\mathrm{Cr}^{6+}$ is transported into cells via sulfate transport mechanisms, taking advantage of the similarities of size and charge between the two ions. Once inside the cell, $\mathrm{Cr}^{6+}$ is reduced to $\mathrm{Cr}^{3+}$ by metabolic agents such as 
Vitamin $\mathrm{C}$, forming hydroxide radicals and other reactive byproducts as a result. These highly reactive products, in turn, are believed to cause DNA damage, which ultimately results in mutation.

\subsubsection{Primers ${ }^{8}$}

Primers are often utilized in conjunction with one of the inhibition methods detailed above — cladding, anodizing, and conversion coatings— to improve the overall capability of the material to resist corrosion. Depending on the application, primers are typically classified as interior or exterior, each having properties tuned to suit its specific need. For example, interior primers are typically not stripped or replaced and must therefore last the lifetime of the material. They are often in contact with liquids such as water, lubricants, and hydraulic fluid and are designed to have greater crosslink densities to reduce the ability of the liquid to permeate and soften (plasticize) the film. In contrast, exterior primers are frequently removed and replaced, so their crosslink densities are typically lower than those of interior primers. Exterior primers must have good adhesion to metal substrates such as aluminum, or to anodized or chromate-converted coatings. Further, exterior primers are often topcoated and so also require good adhesion properties at the primer-coating interface. In general, primer application adds an additional protective barrier against corrosion, however its effectiveness can vary based on the oxygen and water permeability of the film, its adhesion to the substrate, and its resistance to saponification. 


\subsubsection{Primer Oxygen and Water Permeability ${ }^{8}$}

Oxygen, water, and other small molecules can permeate a polymer film even though it has no cracks or imperfections. These molecules transport by various mechanisms between dynamic free volume regions located within the polymer matrix. Given that the free volume increases with temperature at a faster rate above the $T_{g}$, it is important to design coatings with $\mathrm{T}_{\mathrm{g}}$ 's above the temperature at which corrosion protection is desired. Additionally, the rate of crosslinking reactions decreases as the increasing $\mathrm{T}_{\mathrm{g}}$ of the polymer approaches the ambient temperature, therefore this property of the thermoset is limited by environmental conditions for air dry films. This factor can be overcome by increasing the curing temperature (baking). Greater crosslink density reduces pore size, which also reduces the permeability of water and oxygen. Careful consideration is required when designing primers, as modulations in $\mathrm{T}_{\mathrm{g}}$ and crosslink density affect rheological and mechanical properties of the film.

Permeability is also affected by the solubility of oxygen and water. For example, salt groups such as $\mathrm{COO}^{-}$and $\mathrm{NH}_{4}{ }^{+}$can greatly increase water solubility. In contrast, halogenated polymers discourage water solubility. As a result, fluorinated monomers are widely employed in designing polymers for topcoats.

Pigments may be included in the primer formulation to decrease water and oxygen permeability. Oxygen and water molecules cannot diffuse through pigment particles, therefore permeability decreases with increasing pigment volume concentration (PVC) assuming tight wetting of the pigment by the polymer resin. However, if the PVC exceeds a critical volume (critical pigment volume concentration, CPVC), voids can arise in the film through which water and oxygen molecules can diffuse, thereby increasing the permeability of the film. It is important 
to note that permeability influences other aspects of the primer's formulation including the activity of its pigments/additives. For example, if the permeability of the primer is too high, a corrosion inhibitor can leech out of a coating in its early service. In contrast, if the permeability is too low, the corrosion inhibitor will not be able to diffuse through the film to the site(s) of corrosion.

\subsubsection{Primer Adhesion ${ }^{8}$}

The adhesion between the primer and the substrate affects the permeability of the film via an osmotic effect. Water and oxygen that have permeated the film can come into direct contact with the substrate material. If this occurs, all of the necessary elements of corrosion would be present: water, oxygen, conductive impurities on the metal surface - all potentially in contact with both anodic and cathodic regions. Ions produced during the corrosion process gather beneath the film, creating an osmotic cell that draws water through the film to balance the concentration of soluble materials on both sides of the membrane. The osmotic pressure that results from this process can displace the film to create a blister, which increases the area of the surface exposed to corroding elements and thereby increasing the rate of corrosion.

Good wet adhesion reduces the ability of osmotic pressure to displace the film from the substrate. Adhesion can be improved by increasing the surface roughness of the material prior to coating, by increasing the degree of polar functionality of the polymer itself, or by increasing the rigidity of the polymer to prevent the displacement by water of polar and hydrogen bonding interactions. Amine groups have been shown to maximize wet adhesion. They can interact strongly with metal oxides on the surface via hydrogen bonding, however, in general, amine 
functionalized polymers suffer from poor color and gloss retention, and often require topcoats to shield the amine-containing primer from UV radiation.

The viscosity of the primer represents another critical factor affecting its ability to adhere to the substrate. The ability and rate of a fluid to flow in a capillary is dependent on its viscosity. During film formation, the viscosity must remain low enough for the coating to penetrate the microscale pores and crevices. Viscosity increases with crosslink density, thus as the crosslinking reaction proceeds, the capability of the primer to flow into the substrate is depleted. Given this information, it is important to formulate the primer with the tradeoff between viscosity and crosslink density in mind. The pot life of the reaction can be extended through the inclusion of volatile plasticizers. However, this method is accompanied by adverse environmental effects.

\subsubsection{Primer Saponification Resistance ${ }^{8}$}

The process of corrosion generates hydroxide ions at cathodic sites, which can catalyze the hydrolysis of certain functional groups such as esters. Therefore, it can be said that the saponification resistance of a primer depends on its chemistry. For example, epoxy-amine primers exhibit excellent saponification resistance, as these do not contain ester linkages. While amide groups within the backbone of the epoxyamine primer can be hydrolyzed, they are resistant to base-catalyzed hydrolysis.

\subsubsection{Epoxy Primers ${ }^{8}$}

Epoxy primers, in general, are two component $(2 \mathrm{~K})$ formulations. The first component is composed of an epoxide-containing resin in a solvent and may contain additional additives and pigments. The second component is the crosslinking agent, 
typically amine-based, which is dissolved in a solvent. Epoxide resins, in general, are derived from bisphenol A and epichlorohydrin, as in Scheme 1.

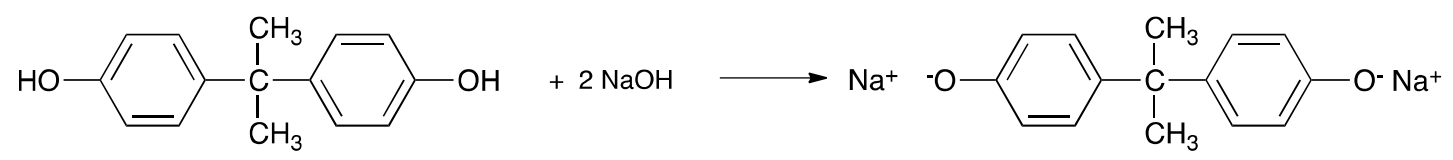<smiles>CC(C)(c1ccc(OCC2CO2)cc1)c1ccc(OCC2CO2)cc1</smiles>

Scheme 1. Typical preparation of a difunctional epoxide monomer.

The resulting difunctional epoxide is then copolymerized with bisphenol A to yield an epoxy resin with epoxide chain end functionality (Scheme 2).

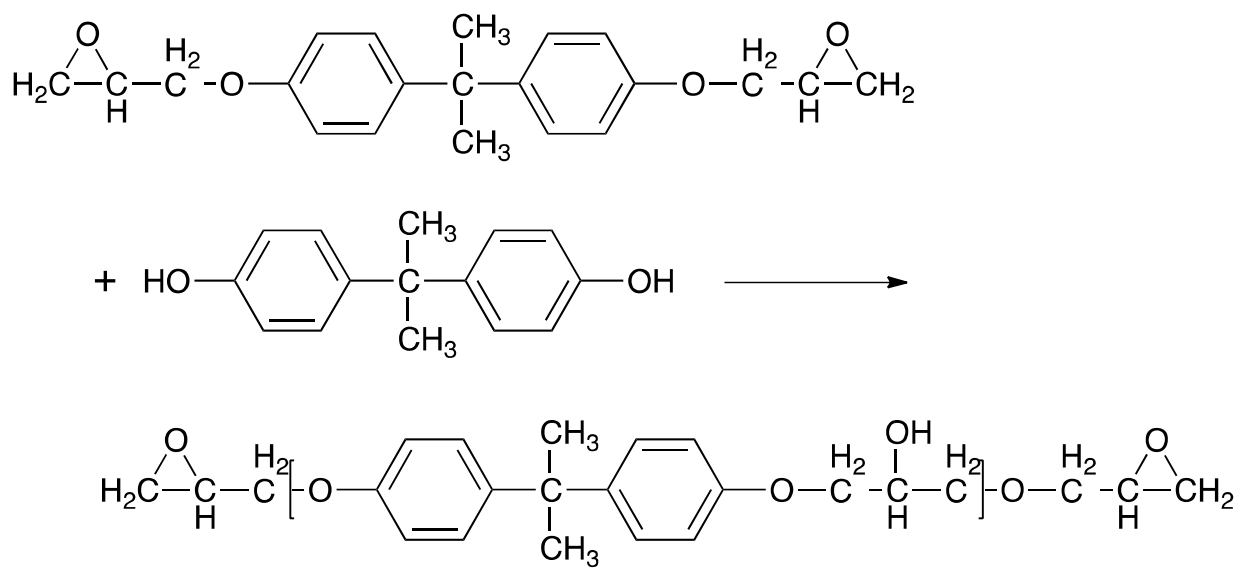

Scheme 2. Preparation of epoxy resin via copolymerization with bisphenol A.

As evident in Scheme 2, the equivalent weight, defined as the number of grams of resin containing one gram equivalent of epoxide groups, is an important parameter that can be tuned to affect the crosslink density of the film. Increasing the copolymer length will decrease the crosslink density, as there will be less epoxide functionality per quantity of resin. However, lower molecular weight polymers can have a plasticizing effect on the bulk material. To circumvent this problem, multiple 
epoxide resins can be mixed to achieve a balance between crosslink density and copolymer molecular weight.

A separate crosslinking agent is required to form the polymer network.

Primary and secondary amines, acids, acid anhydrides, some alcohols, mercaptans, and Lewis acids will react with epoxide functionality to initiate crosslinking under certain conditions. However, amine-based crosslinking agents are preferred because the reaction occurs rapidly at room temperature (Scheme 3).

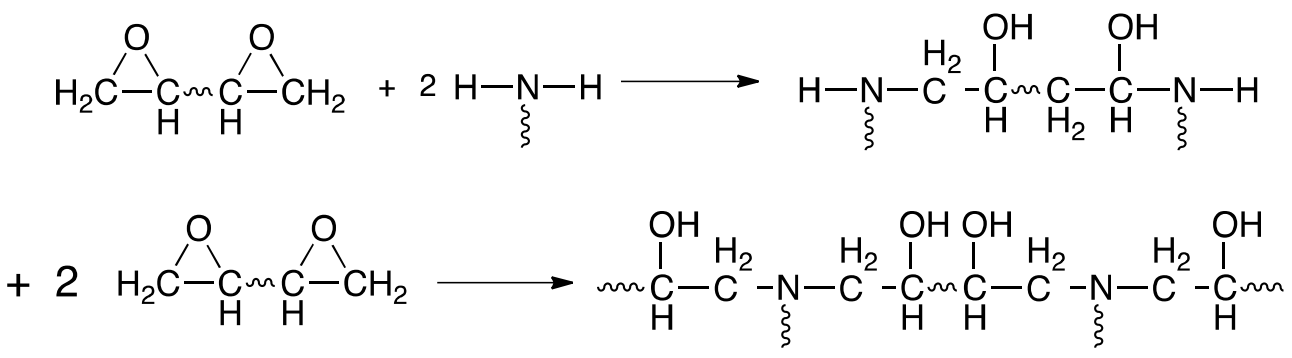

Scheme 3. Generalized epoxy curing reaction.

Like the epoxy resin, the functionality of the crosslinking agent can be manipulated. The higher the functionalities of the resin and crosslinking agent, the higher will be the crosslink density of the resulting film. High crosslink density improves fluid resistance, but can have a detrimental effect on flexibility and impact resistance. The ratio of epoxide to amine equivalents can be adjusted to strike a balance between the properties desired of the system.

Epoxy primers exhibit excellent substrate adhesion due to their hydrogen bonding capability, good recoatability—a layer applied on top of a film will incorporate itself into the existing polymer network through covalent bonding (crosslinking)—excellent fluid resistance, resistance to saponification, and good hardness. The sum of these factors accounts for this primer's ability to improve 
corrosion resistance when applied to a metal substrate. However, epoxy primers suffer the weaknesses of long cure times at room temperature, difficulty in stripping, and poor exterior durability, owing to the high degree of aromaticity, which makes the primer susceptible to UV degradation.

\subsubsection{Chelation Enhanced Fluorescence}

The corrosion of aluminum results in the formation of $\mathrm{Al}^{3+}$ ions via oxidative processes. These ions are then free to participate in chemical reactions, such as metalligand coordination or redox chemistry. Ligands can bind in equivalent proportion, or can form larger complexes in which two or more ligands are bound to the central metal atom. Should the donor species be a fluorophore, the reaction between the $\mathrm{Al}^{3+}$ species and the fluorophore could result in chelation-enhanced fluorescence (CHEF) or chelation-enhanced quenching (CHEQ). ${ }^{9}$ Typically, the binding of metal ions results in a loss of fluorescence intensity.

CHEF defines the process by which an indicator, having both fluorescent and metal-chelating capabilities, binds to a metal ion and subsequently becomes fluorescent. The reaction with the metal ion induces a structural or conformational change (ex. ring-opening) in the indicator molecule, resulting in its activation.
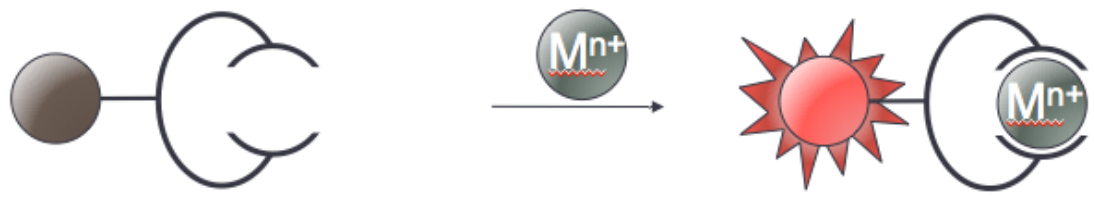

Figure 2. Generalized diagram of CHEF. 


\subsubsection{Schiff Base Chemistry}

The Schiff bases are a subclass of organic compounds containing an imine ( $\mathrm{R}-\mathrm{N}=\mathrm{C})$ bond, where $\mathrm{R}$ is an aryl or alkyl group, not hydrogen. Imine bonds form as the result of nucleophilic attack of a primary amine on the electrophilic carbon of an aldehyde or ketone. ${ }^{10}$ The end result of this reaction is a compound in which the $\mathrm{C}=\mathrm{O}$ double bond has been replaced with $\mathrm{C}=\mathrm{N}$. Mechanistically, the reaction proceeds in two steps. The first step involves nucleophilic attack by the amine on the carbonyl carbon and is similar to the first step of hemiacetal or hemiketal formation. ${ }^{10}$ Second, the nitrogen is deprotonated, and the electrons from this new N-H bond act to eliminate the oxygen, resulting in the displacement of a molecule of water. ${ }^{10}$ Acid can catalyze the reaction. ${ }^{10}$ A generalized reaction mechanism is provided in Scheme 4.

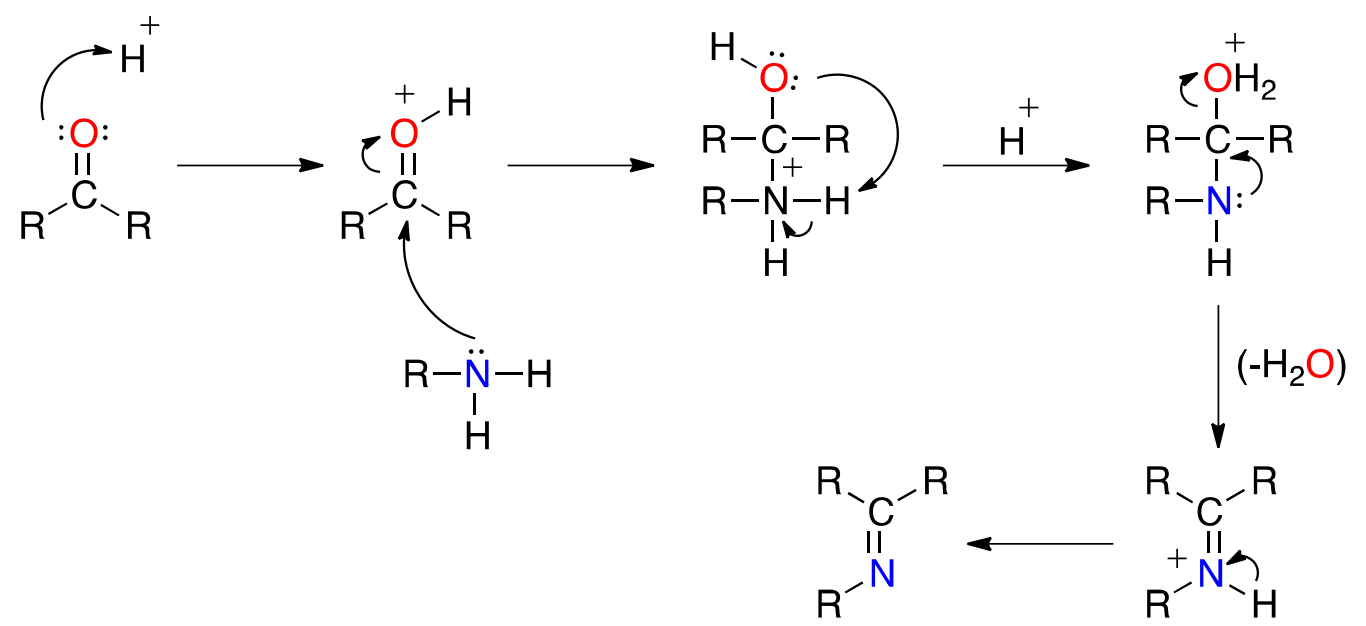

Scheme 4. Generalized mechanism of imine formation. ${ }^{10}$

As an example, the preparation of acetaldimine is shown in Scheme 5. Sodium sulfate acts as a drying agent, driving the reaction by removing water as it is formed. 


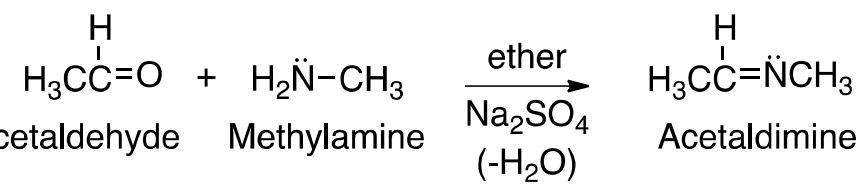

Scheme 5. Preparation of acetaldimine from acetaldehyde and methylamine. ${ }^{10}$

The imine bond is susceptible to hydrolysis under acidic conditions according to Scheme 6, resulting in the reformation of primary amine and carbonyl.

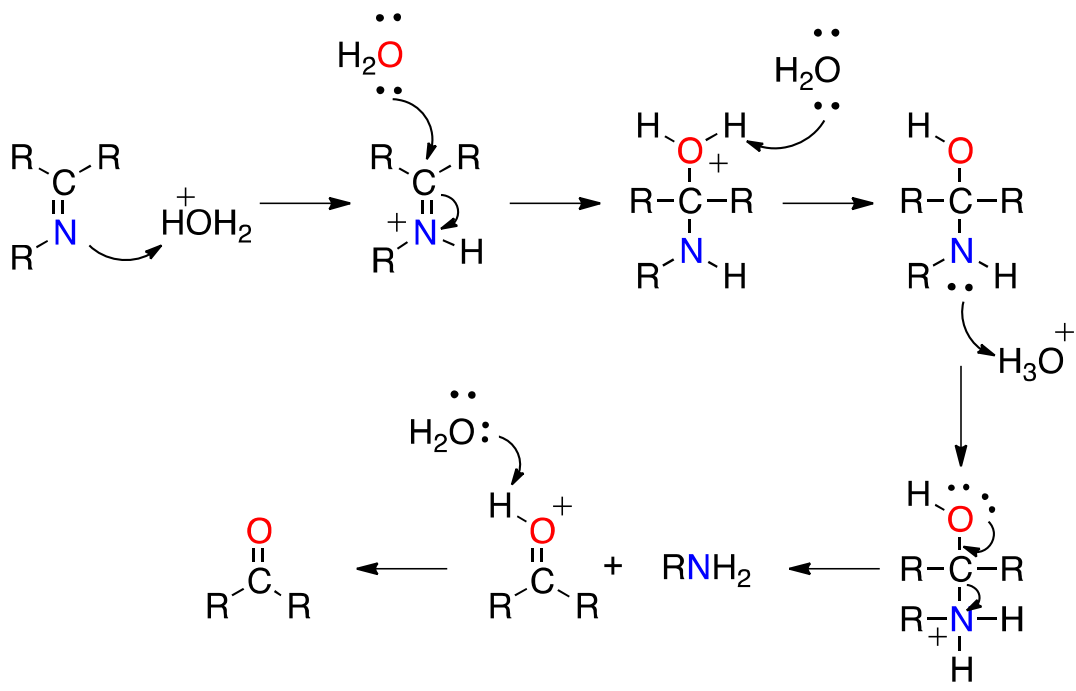

Scheme 6. Generalized mechanism of imine hydrolysis. ${ }^{10}$

\subsection{Prior Work in Literature}

Several fluorescent indicators of aluminum have been reported in the literature, of which, a small subclass have been explored for their capability to detect the corrosion of aluminum.

\subsubsection{Al-binding Fluorophores}

Several indicator molecules capable of reaction with $\mathrm{Al}^{3+}$ ions have been reported. Spectrofluorometric analysis has been conducted on a number of commercially available fluorophores, and novel indicators have been studied as potential indicators 
of corrosion. In an early work, White and Lowe utilized a commercially available indicator, Morin, to determine the concentration of aluminum in solution. ${ }^{11}$

Fluorescence spectroscopy was first employed to obtain an external calibration, and later to determine the concentrations of solutions containing aluminum ions. ${ }^{11}$ Gunduz and coworkers synthesized a novel Schiff base, $N, N^{\prime}$-disalicylidene-1,3diamino-2-hydroxypropane (DSAHP), to quantify a trace amount of aluminum in solution. ${ }^{12}$ The addition of $\mathrm{Al}^{3+}$ was found to increase the fluorescence intensity of DSAHP in ammonium acetate solution, producing an emission maximum at $526 \mathrm{~nm}$ when excited at $410 \mathrm{~nm} .{ }^{12}$ They found that $\mathrm{Al}^{3+}$ could be detected within a limit of quantitation of 0.11-1.62 ppb with a detection limit of $0.07 \mathrm{ppb} .{ }^{12}$ Schiff bases have also been studied by Jiang as potential indicators for $\mathrm{Al}^{3+}$ detection. ${ }^{13}$

\subsubsection{Indicators for the Detection of Corrosion}

Fluorescent indicators have been evaluated with respect to their ability to be used in the detection of corrosion. In situ detection of corrosion at the substrate/coating interface can be achieved by incorporating indicator molecules into the coating formulation. For example, Sibi and Zong have reported a method of corrosion detection for aluminum substrates. ${ }^{14}$ They applied a primer coating containing lumogallion to an aluminum substrate, submerged the coated aluminum substrate into an electrolyte solution, and monitored its corrosion by following the release of the Allumogallion complex into solution via fluorescence spectroscopy. ${ }^{14}$ Fluorescence microscopy of the coated substrates demonstrated the capability of fluorescent indicators to identify the localized zones of corrosion. However, in a later study, 
Chambers and colleagues argued that interferences limited the effectiveness of lumogallion to detect corrosion in complex coated systems. ${ }^{15,16}$

Ming et. Al. showed that the early detection of corrosion could be detected by following the release of ferric ions into a polymer film. ${ }^{17}$ To this end, they dispersed a spirocyclic rhodamine compound into an epoxy/polyamide coating prior to application to the metal surface. They concluded that the early onset of corrosion could be detected in steel substrates before any visual sign of corrosion appears.

\subsubsection{Non-fluorescent Methods of Corrosion Detection}

A variety of non-fluorescent corrosion detection methods have been evaluated in the literature. Electrochemical impedance spectroscopy (EIS) has been shown to be capable of successfully evaluating the properties of polymer-coated metals and their changes during exposure to corrosive environments. ${ }^{22}$ Early work by Menges and Schneider identified the potential advantages of this technique, and later Beaunier $e t$ al. developed the equivalent circuit used for the bulk of corrosion related experiments that utilize EIS (Scheme 7). ${ }^{22}$

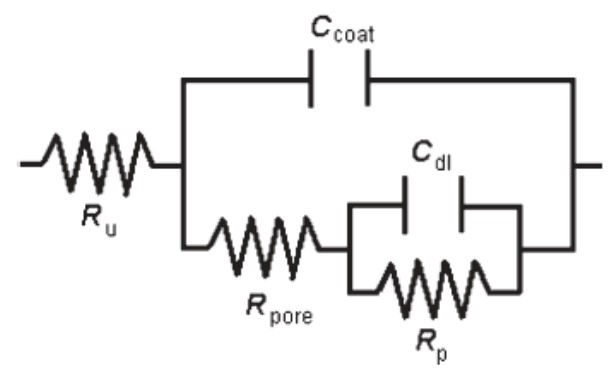

Scheme 7. Equivalent circuit model used to describe metals with organic coatings. ${ }^{22}$ In Beaunier's model, the water uptake of the coating can be estimated from the time dependence of the coating capacitance, $\mathrm{C}_{\mathrm{c}}{ }^{22}$ As corrosion proceeds and the coating is damaged, a decreased coating resistivity, $\rho$, is observed, corresponding to a 
similar decrease in pore resistance, $\mathrm{R}_{\mathrm{po}}{ }^{22}$ Eventually, disbonding of the coating occurs and can be monitored by following changes in the polarization resistance, $R_{p}$, and the capacitance, $\mathrm{C}_{\mathrm{dl}}$, which are both related to the delaminated area, $A_{d}$, or the delamination ratio, D. ${ }^{22}$ It has been demonstrated that a complete analysis of the performance of a coating system is possible based on EIS data collected at relatively high frequencies. $^{22}$

Similarly, pulsed phase thermography (PPT) is a non-destructive tool that can be used to analyze inhomogeneities and defects in the films of coated metals. ${ }^{23}$ Recent work presented in the literature has demonstrated the potential of PPT to detect the propagation of corrosion under coatings. ${ }^{23}$ Although non-quantitative, PPT has demonstrated the capability to detect corrosion and delamination on steel and galvanized steel panels coated with organic paint. ${ }^{23}$

Fiber optic sensors have been used for the detection of corrosion at a large scale. ${ }^{24}$ Fuhr and Hutson embedded multiple-parameter sensing fiber optic sensors into steel reinforced concrete samples. ${ }^{24} \mathrm{~A}$ broadband of light was transmitted through the fiber to the steel surface and either collected by a second fiber or reflected back to the first. ${ }^{24}$ Color shifts induced by the presence of corrosion were measured. ${ }^{24}$ In this application, corrosion is indicated by damage to the fiber, which is assumed to result from the occurrence of corrosion within the material. ${ }^{24}$ 


\section{Chapter 3: Methods and Materials}

\subsection{Reagents}

All reagents were obtained from commercially available sources and used without further purification. Rhodamine-acetone (RAc) was synthesized as previously reported. ${ }^{18}$

\subsection{Synthesis of Schiff Bases}

All Schiff bases were prepared according to a similar process. For all, the amino reagent was dissolved in ethanol and heated to reflux. Next, a volume of salicylaldehyde was added dropwise. These reactions resulted in the formation of insoluble solids, which were isolated via vacuum filtration. The synthesis of salicylaldehyde o-aminophenol (SOAP) will be provided as an example.

\subsubsection{Synthesis of Salicylaldehyde o-Aminophenol}

SOAP was prepared via a one-pot reaction. o-Aminophenol (436.3 mg, $3.999 \mathrm{mmol})$ was dissolved with heating in ethanol (95\%). To this solution, a volume of salicylaldehyde $(0.42 \mathrm{~mL}, 488.48 \mathrm{mg}, 4.0 \mathrm{mmol})$ was added dropwise. The reaction mixture was heated to reflux and stirred for a period of 1.5 hours, after which the reaction was cooled in an ice bath. A deep red precipitate formed and was collected via vacuum filtration. Yield: $758.7 \mathrm{mg}, 3.558 \mathrm{mmol}(89 \%) .{ }^{1} \mathrm{H}$ NMR analysis of the product agreed with the spectrum reported in the literature. ${ }^{26}$ 


\subsection{Synthesis of Rhodamine-salicylaldehde (RSA)}

RSA was synthesized in two steps from Rhodamine B base. In the first step, Rhodamine B base (4.21 g, $9.51 \mathrm{mmol})$ was dissolved in ethanol ( 180 mL, 95\%). A twenty-fold molar excess of hydrazine hydrate was added via syringe and the reaction mixture was stirred overnight. After solvent removal, the residue was redissolved in dichloromethane. The dichloromethane solution was washed with distilled water and dried over anhydrous sodium sulfate. Following removal of the dichloromethane, the obtained residue was purified by column chromatography (eluted with 90:10 $\left.\mathrm{CH}_{2} \mathrm{Cl}_{2} / \mathrm{MeOH}\right)$ to give an off-white powder. Yield: $3.14 \mathrm{~g}, 6.88 \mathrm{mmol}(72 \%)$.

In the second step, $0.73 \mathrm{~g}(1.59 \mathrm{mmol})$ of the product obtained in step one was dissolved in ethanol ( $70 \mathrm{~mL}, 95 \%)$. A two-fold molar excess of salicylaldehyde and a catalytic amount of glacial acetic acid were added via pipette and the reaction mixture was heated at reflux overnight. The resulting precipitate was collected by vacuum filtration and allowed to dry overnight to yield RSA, $0.55 \mathrm{~g}, 0.98 \mathrm{mmol}$

(61\%). ${ }^{1} \mathrm{H}$ NMR (300 MHz, ACN-d 3 ) $1.13(3 \mathrm{H}, \mathrm{d}), 3.36$ (2H, q), 6.40 (1H, dd), 6.46 $(2 \mathrm{H}, \mathrm{s}), 6.51(1 \mathrm{H}, \mathrm{d}), 6.82(1 \mathrm{H}, \mathrm{d}), 6.86(1 \mathrm{H}, \mathrm{t}), 7.14(2 \mathrm{H}, \mathrm{d}), 7.20(2 \mathrm{H}, \mathrm{d}), 7.61(1 \mathrm{H}$, m), $7.94(1 \mathrm{H}, \mathrm{d}), 9.20(1 \mathrm{H}, \mathrm{s}), 10.80(1 \mathrm{H}, \mathrm{s})$.

\subsection{Synthesis of RSA-Al Complex}

The RSA-Al complex was prepared by first dissolving RSA (103 mg, $0.0184 \mathrm{mmol}$ ) in toluene $(\sim 5 \mathrm{~mL})$. Next, $200 \mu \mathrm{L}$ (208 mg, $1.72 \mathrm{mmol})$ of diethylaluminum chloride was added via purged syringe. Upon addition, a brilliant violet precipitate formed that was isolated by vacuum filtration. The obtained precipitate was washed with toluene and allowed to dry overnight. Yield: $98 \mathrm{mg}, 0.016 \mathrm{mmol}(95 \%)$. 


\subsection{Characterization}

\subsection{1 ${ }^{1} \mathrm{H}$ NMR Spectroscopy}

${ }^{1} \mathrm{H}$ NMR spectra were collected on a Bruker UltraShield $300 \mathrm{MHz}$ NMR

spectrometer. Solutions $(0.27 \mathrm{M})$ of salicylaldehyde and Rhodamine salicylaldehyde

(RSA) were prepared by dissolving both chemicals in acetonitrile- $\mathrm{d}_{3}$. Then, equimolar volumes of $\mathrm{DCl}$ in $\mathrm{D}_{2} \mathrm{O}$ or $\mathrm{Al}\left(\mathrm{NO}_{3}\right)_{3}$ in acetonitrile- $\mathrm{d}_{3}$ were added via micropipette to both solutions.

\subsection{2 ${ }^{27} \mathrm{Al}$ NMR Spectroscopy}

${ }^{27} \mathrm{Al}$ spectra were collected on a Bruker UtraShield $300 \mathrm{MHz}$ NMR spectrometer. A quantity of $20 \mathrm{mg}$ of $\mathrm{Al}\left(\mathrm{NO}_{3}\right)_{3}$ was dissolved in a 50:50 mixture of toluene/methanol$\mathrm{d} 4$ and its spectra was obtained. A coaxial NMR tube was employed, with $\mathrm{Al}\left(\mathrm{NO}_{3}\right)_{3}$ in deuterated water serving as the external standard. A titration was performed by preparing 3:2, 1:1, and 2:3 mole equivalent solutions of $\mathrm{Al}\left(\mathrm{NO}_{3}\right)_{3}$ to $\mathbf{R S A}$ and comparing the relative integration values of their hydrated $\mathrm{Al}^{3+}$ peaks.

\subsubsection{Fluorescence and UV-Vis Analysis of RSA}

Fluorescence emission spectra of RSA in solution were obtained with a Jasco FP6500 fluorescence spectrometer with an excitation wavelength of $512 \mathrm{~nm}$. Absorbance spectra were collected with a Jasco V-550 UV/Vis spectrophotometer. A solution $(100 \mu \mathrm{M})$ of RSA was prepared by dissolving the fluorescent indicator in acetonitrile. This solution was diluted to $10 \mu \mathrm{M}$ in the cuvette. Equimolar volumes of $\mathrm{Al}\left(\mathrm{NO}_{3}\right)_{3}, \mathrm{FeCl}_{2}$ and $\mathrm{CuSO}_{4}$, in acetonitrile or $200 \mu \mathrm{M} \mathrm{HCl}$ in distilled water were 
added to obtain the ligand's excitation and emission spectra under different conditions.

\subsubsection{Fluorescence Imaging}

Coated glass slides and aluminum panels were scanned with the Typhoon Trio+ fluorescence imager. The $488 \mathrm{~nm}$ and $532 \mathrm{~nm}$ emission sources were used at a PMT setting of $500 \mathrm{~V}$. The scan produced grayscale images, with the darkest pixels corresponding to the areas on the samples where the fluorescence intensity was greatest. The obtained images were analyzed with ImageQuant to determine (1) the average intensity of the sample over a predefined area and (2) the area of pixels, within a specified boundary, that had an intensity greater than the threshold value of 100,000 counts. The area with intensity above the threshold was plotted vs. time in order to assess the degree of corrosion across the lifetime of the experiment.

\subsection{Epoxy Coating Formulation}

The two-component epoxy was prepared according to the formulation outlined in Appendix A. Each component was prepared by weighing the appropriate quantities of resins, additives, and solvents and mixing with a Thinky Mixer AR-100 planetary centrifugal mixer for one minute at its maximum RPM setting. The A and B components were then combined and mixed. An induction period of 30 min was allotted before the coatings were applied. 


\subsection{Preparation of Coated Glass Slides}

The surfaces of glass microscope slides $(75 \mathrm{~mm}$ x $25 \mathrm{~mm}$ ) were washed with acetone to remove any organic residue. Epoxy coatings were then applied utilizing a $75 \mu \mathrm{m}$ (wet film thickness) drawdown bar. When appropriate, fluorescent indicators were added to the epoxy formulations at $1 \% \mathrm{w} / \mathrm{w}$ (dry) prior to application. The indicators were dissolved in a small quantity of solvent, typically toluene or ethanol, to aid in dispersion. The coated slides were cured at $70^{\circ} \mathrm{C}$ for 24 hours prior to testing.

\subsection{Preparation of Coated Aluminum Panels}

Al-3003 H14 panels (76 mm x $152 \mathrm{~mm})$ and Al-2024 T3 coupons (25 mm x $102 \mathrm{~mm})$ were abraded with sandpaper to remove aluminum oxides and rinsed with hexanes prior to coating application. A 3 mil (wet film thickness) drawdown bar was used to apply the epoxy coatings. When appropriate, RSA was added to the epoxy formulations at $1 \% \mathrm{w} / \mathrm{w}$ (dry) prior to application. The indicator was dissolved in a small quantity of toluene to aid in dispersion. The coated panels were cured at $70^{\circ} \mathrm{C}$ for 24 hours prior to testing.

\subsection{Immersion Corrosion}

Coated Al-2024 T3 aluminum coupons were submerged in a 3\% w/v solution of $\mathrm{NaCl}$. The coupons were soaked for 24 hours, removed from the solution and dried for $1 \mathrm{hr}$ at $70^{\circ} \mathrm{C}$, rinsed with deionized water, and imaged with the Typhoon Trio+. The green $(532 \mathrm{~nm})$ excitation source was used with a PMT setting of $500 \mathrm{~V}$ and a $580 \mathrm{~nm}$ emission filer. This cycle was repeated daily over a period of 3 weeks. 


\subsection{Salt Spray Corrosion}

Coated Al-2024 T3 aluminum panels were loaded into the Q-Fog Cyclic Corrosion Tester. The instrument was configured according to the prohesion standard (ASTM G85). The panels were corrode for 24 hours, removed from the chamber and dried at $70^{\circ} \mathrm{C}$ for $1 \mathrm{hr}$, rinsed with deionized water, and imaged with the Typhoon Trio+. The green $532 \mathrm{~nm}$ excitation source was used with a PMT setting of $500 \mathrm{~V}$ and a $580 \mathrm{~nm}$ emission filer. This cycle was repeated daily over a period of 360 hours.

\subsection{Spray-on Corrosion Test}

Coated Al-2024 T3 aluminum panels that had been corroded for 3000 hours with the Q-Fog Cyclic Corrosion Tester were sprayed with a $1 \%$ w/v solution of RSA in toluene and allowed to dry at room temperature. The panels were images with the Typhoon Trio+ prior to spray application, and again after the RSA solution had dried. The green $532 \mathrm{~nm}$ excitation source was used with a PMT setting of $500 \mathrm{~V}$ and a 580 $\mathrm{nm}$ emission filer. 


\section{Chapter 4: Results and Discussion}

\subsection{Evaluation of Commercial Ligands}

The initial phase of this study focused on the evaluation of the proposed Al-sensitive fluorophores in solution and in epoxy coatings on glass substrates. Several fluorophores were selected and evaluated based upon literature reports of their efficacy as proficient indicators of aluminum ions in solution. These indicators have been reported to show large increases in fluorescence intensity in the presence of small concentrations of aluminum ions (part-per-million to part-per-billion detection limits). ${ }^{9,16}$ For example, the commercial indicator Lumogallion fluoresced bright orange under UV light following the addition of aluminum nitrate (Figure 3).
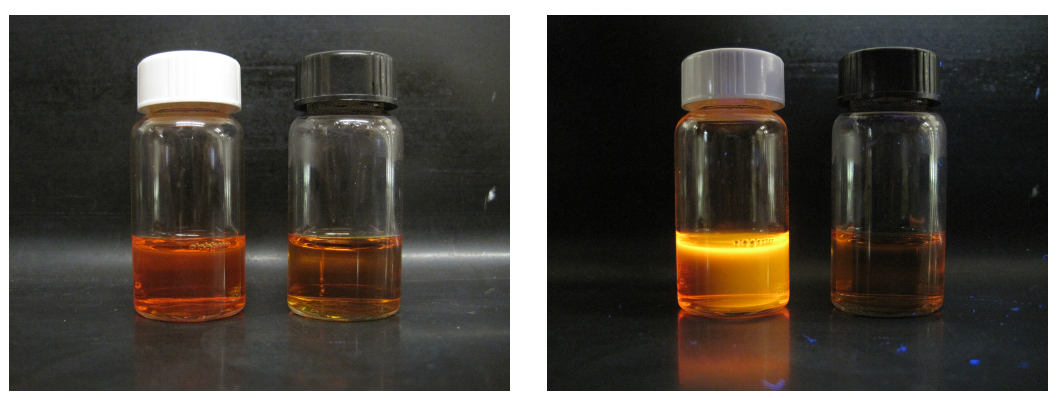

Figure 3. Fluorescence activation of Lumogallion following the addition of aluminum nitrate.

For a fluorophore to be considered a viable indicator of corrosion, it must be compatible with the solvent-based epoxy system, must not leech into the $\mathrm{NaCl}$ solution in which it is submerged for immersion corrosion testing, must be compatible with the fluorescence imager (i.e. its excitation wavelength must be near those capable of the instrument's three laser light sources and its emission must fall within the bandwidth limits of the Typhoon's emission filters), and must be sensitive 
enough to detect trace amounts of aluminum so that corrosion may be discovered in its early stages.

The commercially available ligands shown in Figure 4 were evaluated for potential use as corrosion indicators. Compounds 1-3 have been studied in coatings on aluminum substrates. ${ }^{9,16}$<smiles>O=c1c(O)c(-c2ccc(O)cc2O)oc2cc(O)cc(O)c12</smiles><smiles>[Mg][Mg]</smiles>

1<smiles>O=S(=O)(O)c1ccc(O)c2ncccc12</smiles>

8-HQS 2<smiles>O=S(=O)(O)c1cc(Cl)cc(N=Nc2ccc(O)cc2O)c1O</smiles>

Lumogallion

Figure 4. Commercial ligands evaluated as potential corrosion indicators.

To determine their compatibility with the epoxy system, the solubility of

Compounds 1-3 in selected solvents was evaluated by depositing a small amount (1-5 $\mathrm{mg}$ ) of each into a $1 \mathrm{~mL}$ volume of liquid and observing their degree of solubility. An empirical summary of the solubilities of these fluorescent indicators is provided in Table 3.

Table 3. Solubilities of commercial ligands in selected solvents.

\begin{tabular}{|c|c|c|c|c|}
\hline & \multicolumn{4}{|c|}{ Solubility in } \\
\hline Compound & Water & Ethanol & Acetonitrile & Toluene \\
\hline $\mathbf{1}$ & SS & HS & SS & I \\
\hline $\mathbf{2}$ & HS & SS & I & I \\
\hline $\mathbf{3}$ & HS & SS & I & I \\
\hline
\end{tabular}

*HS: highly soluble; SS: slightly soluble; I: insoluble.

As shown in Table 3, the three commercial indicators are solvated by polar and polar-protic solvents primarily, and do not dissolve in the more hydrophobic organic solvents acetonitrile and toluene. Looking at the structures of these molecules, it is clear why this is the case-compounds $\mathbf{1}$ and $\mathbf{3}$ especially are rich 
with hydroxyl functionality, enabling these molecules to interact with water and other protic solvents via hydrogen bonding. Unfortunately, their insolubility in hydrophobic solvents makes their dispersion in the solvent-based epoxy difficult.

The excitation and emission wavelengths of the commercial indicators were determined using standard fluorescence spectroscopy. Excitation and emission spectra were collected in a recursive process, whereby the emission maximum would be used to select the excitation wavelength and this optimized parameter would in turn be employed to determine the ideal emission wavelength. The excitation wavelengths of these compounds were found to be incompatible with the instrumentation to be used for our high throughput method. The Typhoon Trio+ Variable Mode Fluorescence Imager is capable of three excitation wavelengths; 488, 532, and $633 \mathrm{~nm}$. As shown in Table 5, the excitation wavelengths for Morin and HQS are too low to be useful on the Typhoon imager.

Table 4. Excitation wavelengths found for commercial indicators.

\begin{tabular}{|l|c|c|}
\hline \multicolumn{1}{|c|}{ Fluorophore } & Excitation $\boldsymbol{\lambda}(\mathbf{n m})$ & Emission $\boldsymbol{\lambda}(\mathbf{n m})$ \\
\hline Morin & 418 & 498 \\
\hline HQS & 367 & 495 \\
\hline Lumogallion & 502 & 575 \\
\hline
\end{tabular}

Epoxy coatings composed of EPON Resin 1001-CX-75 and EPICURE Curing Agent and containing $1 \% \mathrm{w} / \mathrm{w}$ indicator were spread on glass microscope slides. Following a 24-hour curing period, the resulting films were imaged using an optical microscope and the Typhoon confocal imager. Optical microscope images clearly show the presence of dye aggregates within the coatings, as shown in Figure 5. Aggregation of dye molecules is undesirable for this application. 


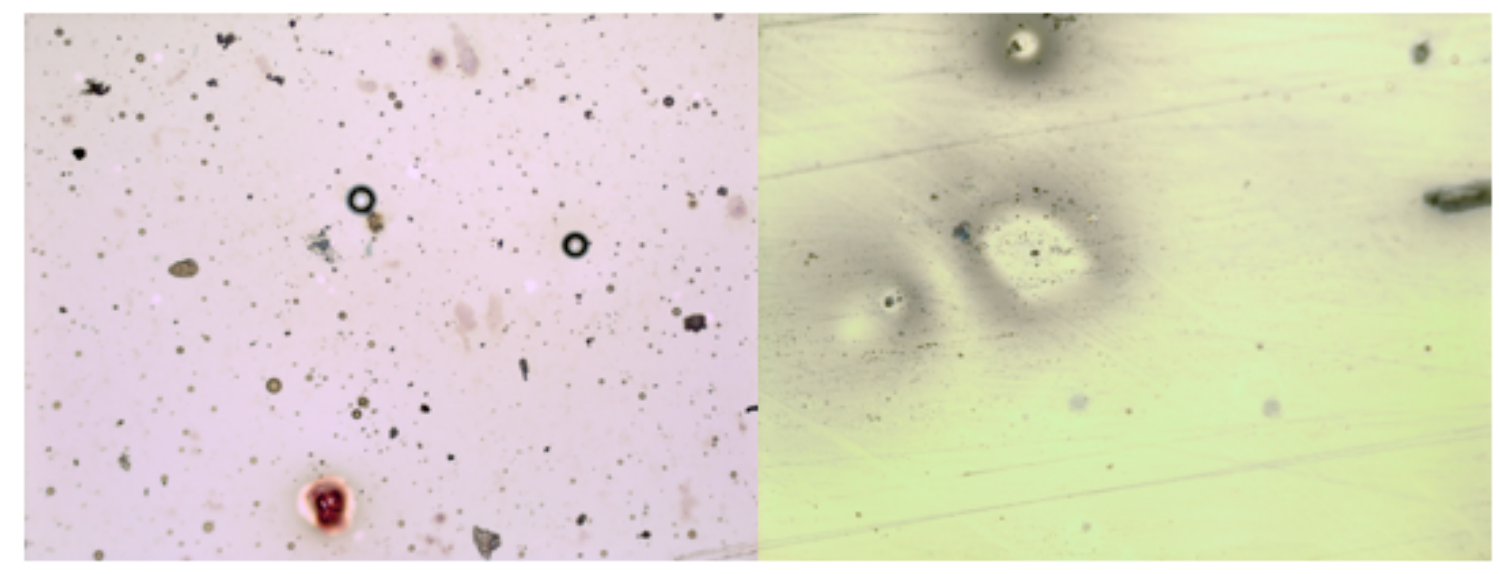

Figure 5. Optical microscope image of Morin (left) and 8-HQS (right) showing poorly dispersed dye aggregates and epoxy coating imperfections-the result of the solvents utilized and poor solubility in the dry film.

In addition, attempts to image epoxy coatings containing these fluorophores, with the exception of 8-HQS, did not show a significant difference in the presence and absence of aluminum ions, as shown in Table 4. The coated microscope slides were submerged in a $3 \% \mathrm{w} / \mathrm{v} \mathrm{NaCl}$ solution. Two different conditions were investigated; $532 \mathrm{~nm}$ excitation with $580 \mathrm{~nm}$ emission filter and $488 \mathrm{~nm}$ excitation with a $520 \mathrm{~nm}$ emission filter. Loss of intensity for the Lumogallion-containing epoxy coatings could be attributed to loss of water-soluble Lumogallion into the aqueous solution. The increase in intensity for 8-HQS when imaged at $532 \mathrm{~nm}$ excitation is unusual and can not be explained.

Table 5. Summary of coated glass slide immersion tests.

\begin{tabular}{|c|c|c|}
\hline Indicator & increase (532nm) & increase (488nm) \\
\hline Morin (5-6) & $12 \%$ & $0 \%$ \\
\hline 8-HQS (9-10) & $66 \%$ & $19 \%$ \\
\hline Lumogallion (7-8) & $-38 \%$ & $-2 \%$ \\
\hline
\end{tabular}

The insolubility of Compounds $\mathbf{1 - 3}$ in organic solvents in combination with the incompatibility of these indicators with the Typhoon confocal imager makes these 
molecules poor choices for indicators of corrosion in our system. As such, they were eliminated from consideration for long-term corrosion studies.

\subsection{Schiff Base Ligands}

Following our evaluation of the commercial indicators Morin, 8-HQS, and Lumogallion, the next phase of the study focused on Schiff base-containing fluorophores (Figure 6). The Schiff base salicylaldehyde o-aminophenol (SOAP) is known to complex with aluminum ions in solution. ${ }^{19}$

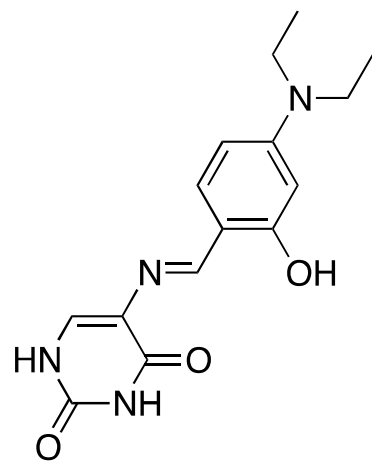

4

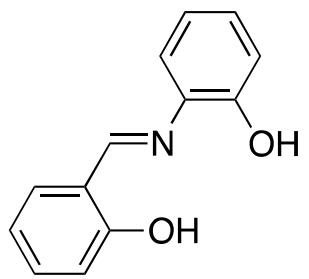

5

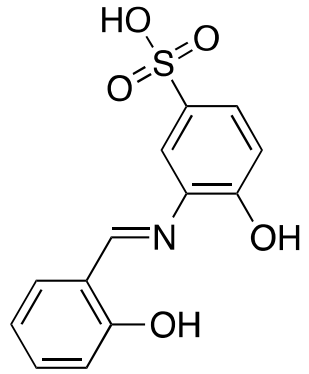

6

Figure 6. Schiff bases evaluated as potential indicators of corrosion.

Unlike the commercial indicators, the Schiff base indicators evaluated had to

be synthesized. The synthesis of these compounds followed a one-step facile condensation mechanism in which an aldehyde was reacted with an amine to produce an imine $(\mathrm{C}=\mathrm{N}-\mathrm{R})$ bond. The preparation of SOAP, Compound $\mathbf{5}$, is presented in Scheme 8 .

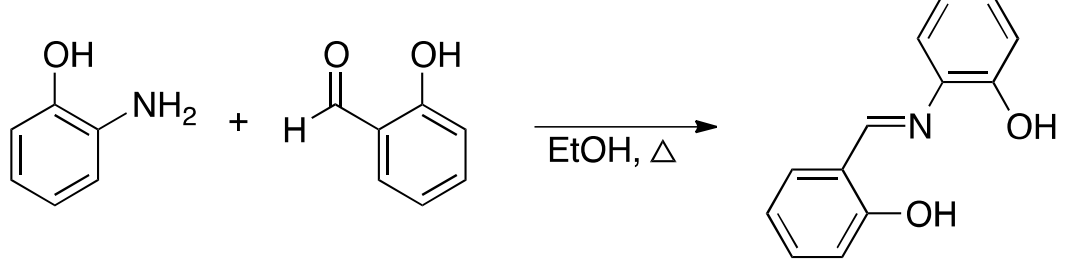

Scheme 8. Synthesis of salicylaldehyde o-aminophenol. 
Fluorescence spectroscopy of compounds $\mathbf{4}$ - $\mathbf{6}$ confirmed the high sensitivity of these compounds towards aluminum ions. All three were capable of detecting aluminum in the ppb range. The fluorescence response of SOAP with increasing amounts of aluminum nitrate is shown as an example in Figure 7. Concentrations of aluminum nitrate as low as $107 \mathrm{ppb}$ could be distinguished from the background intensity.

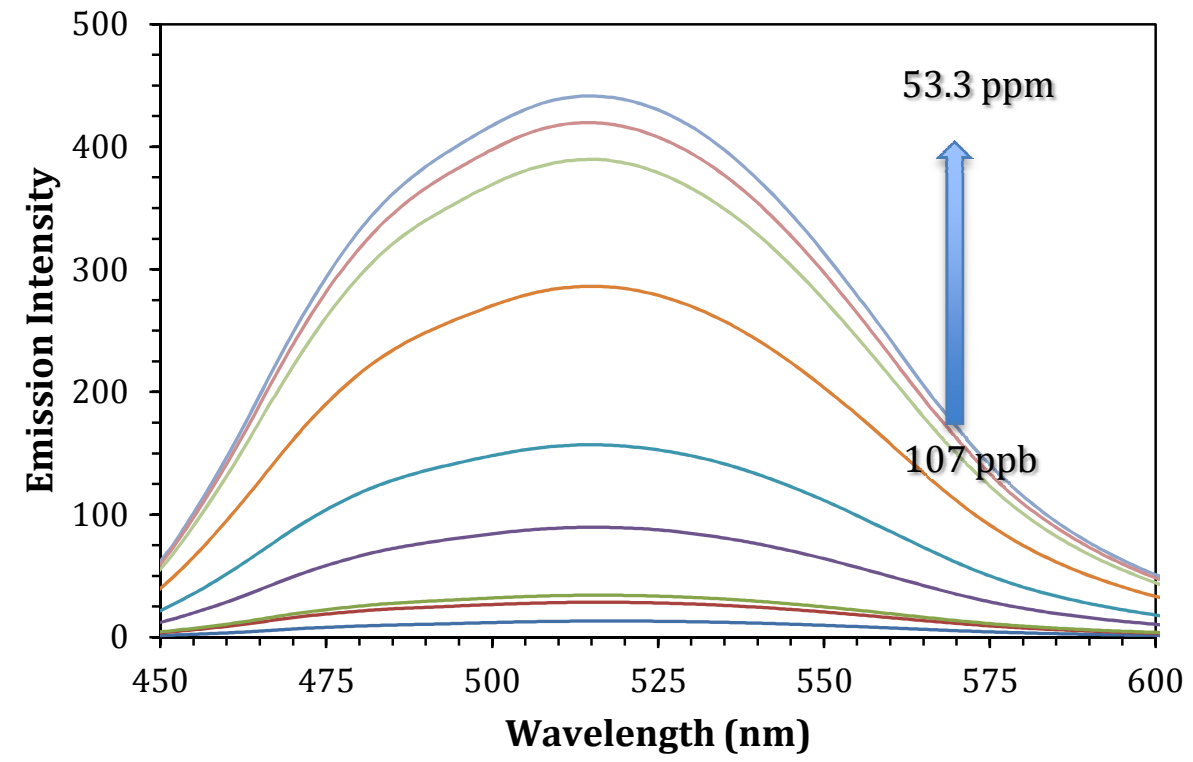

Figure 7. Fluorescence response of a $50 \mu \mathrm{M}$ solution of Compound 5 in ethanol with increasing amounts of aluminum nitrate.

Although many of these compounds show incredible sensitivity for aluminum ions, they proved to be poor candidates for our studies in epoxy coatings. Many of our initial candidates are unstable in solution and degrade significantly in less than 24 hrs. For example, compound $\mathbf{5}$ showed a strong fluorescence in the presence of aluminum ions, but the response of the aluminum complex decreased by approximately $60 \%$ after an hour in solution (Figure 8). 


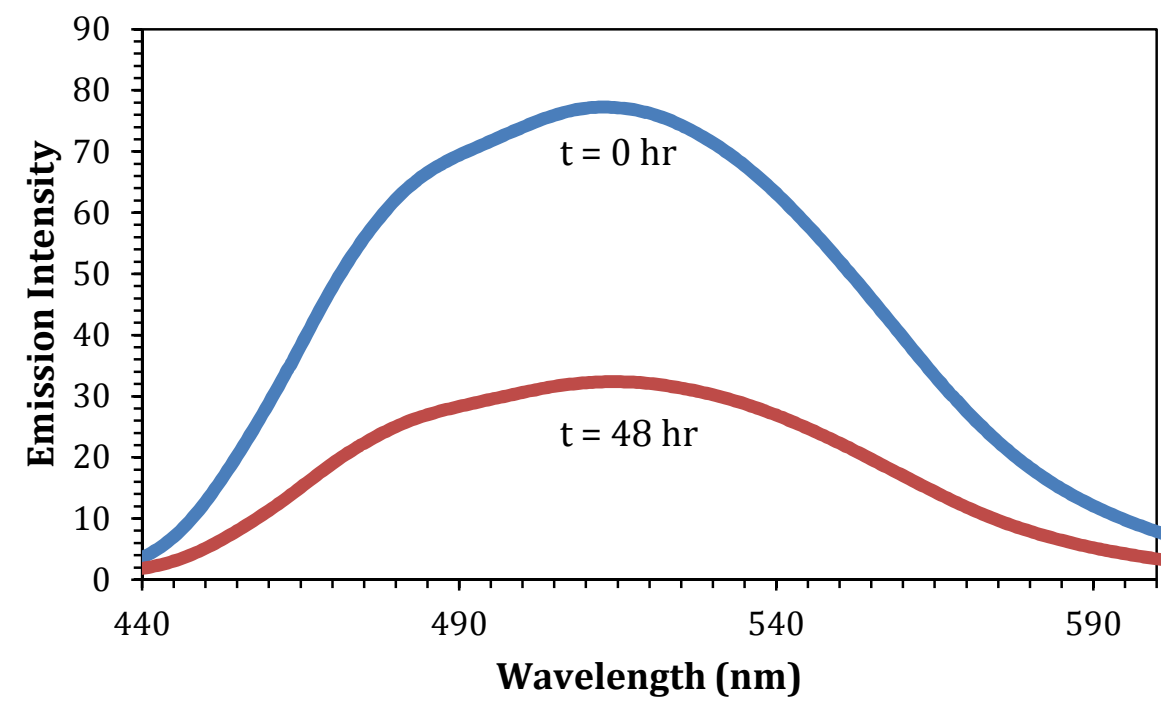

Figure 8. Loss of fluorescence intensity of a $50 \mu \mathrm{M}$ solution Compound 5 with an equimolar amount of aluminum nitrate in ethanol after 48 hours in solution-the result of imine bond hydrolysis.

Each fluorophore proved to be unstable in aqueous solution over the long period of time required for the proposed corrosion testing. This is likely due to the hydrolysable imine group in these ligands. In addition, the aluminum compounds appear to photodegrade under prolonged exposure to light. Both of these properties are unacceptable, as the ultimate goal of this project is to use these compounds to monitor corrosion over extended time periods. Regardless of the mechanism of deactivation, the Schiff base derived fluorophores are not practical choices for this application.

\subsection{Rhodamine Derivatives}

Rhodamine-based fluorophores (Figure 9) were evaluated as potential indicators of corrosion based on their known capability to bind transition metal ions, the fact that their excitation and emission wavelengths fall within the range of the Typhoon 
imager, and their greater degree of hydrophobicity which enables their dispersion in our solvent borne epoxy system.

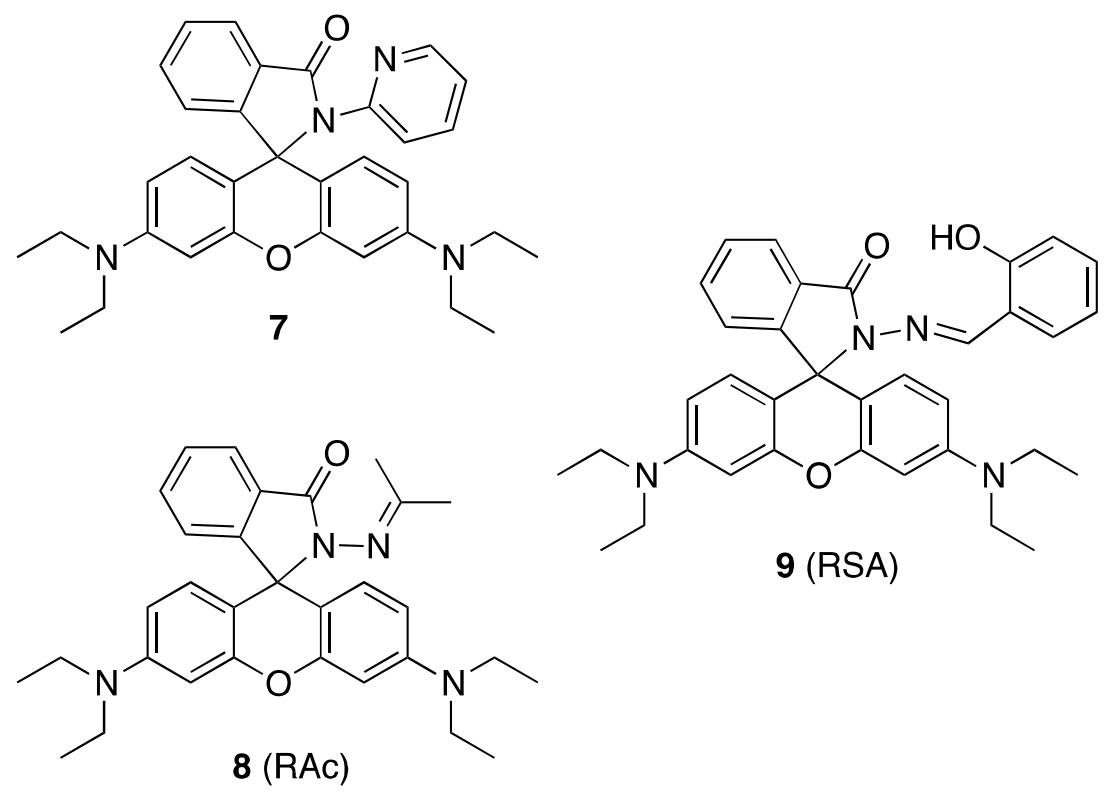

Figure 9. Rhodamine ligands evaluated for their efficacy as indicators of the corrosion of aluminum substrates.

Compound 7 has been investigated for its capability to bind transition metal cations. ${ }^{18}$ Zang and coworkers found that this ligand became activated following the addition of iron, mercury, and lead ions to an acetonitrile solution containing the fluorophore. ${ }^{18} \mathrm{UV}$-Vis, fluorescence, IR, and ${ }^{1} \mathrm{H}$ NMR spectroscopy were used to investigate the binding mechanism, leading to the conclusion that the ligand formed a 2:1 complex with a metal ion, where the binding sites $(\mathrm{N}$ and $\mathrm{O})$ within each of Compound 7 form a six-membered chelate ring with the cation. ${ }^{18}$

Augustyniak et al. incorporated $\mathbf{8}$ into epoxy coatings on steel substrates to evaluate the efficacy of this indicator to detect corrosion. ${ }^{17}$ Concentrations as low as $0.5 \mathrm{wt} \%$ were sufficient to detect the corrosion of iron at the early stages. ${ }^{17}$ Coated 
steel coupons were imaged with fluorescence microscopy, which revealed areas of intense fluorescence around regions that had been scribed and had begun to corrode. ${ }^{17}$

Compounds 8 and 9 were investigated in this study. They are synthesized according to Scheme 8 . The preparation follows a two-step synthesis by which a hydrazine derivative of Rhodamine B is formed and isolated. Mechanistically, the first step of this reaction proceeds through a carbonium intermediate, shown in Scheme $9 .{ }^{20}$ Rotation around the carbon-nitrogen bond of the hydrazino group is severely hindered due to the 9 -aryl ring. ${ }^{20}$ As such, the subsequent ring closure occurs via the nucleophilic attack of the secondary amine, yielding the hydrazide shown above. $^{20}$
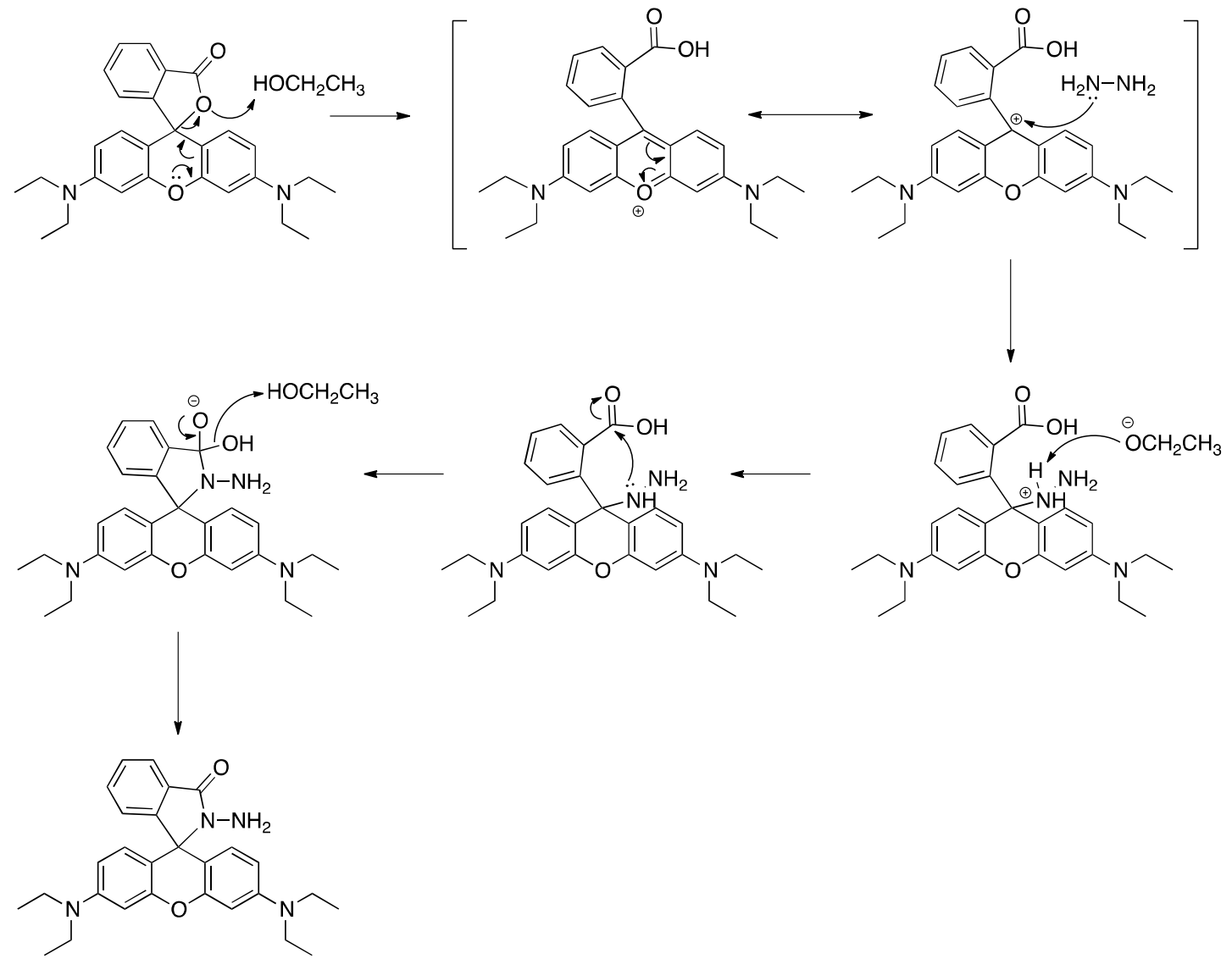

Scheme 9. Proposed mechanism for the formation of rhodamine-hydradize in ethanol. $^{20}$ 
In the second step, the hydrazide compound is reacted with either acetone or salicylaldehyde to produce Rhodamine-acetone (RAc) and Rhodaminesalicylaldehyde (RSA) respectively.

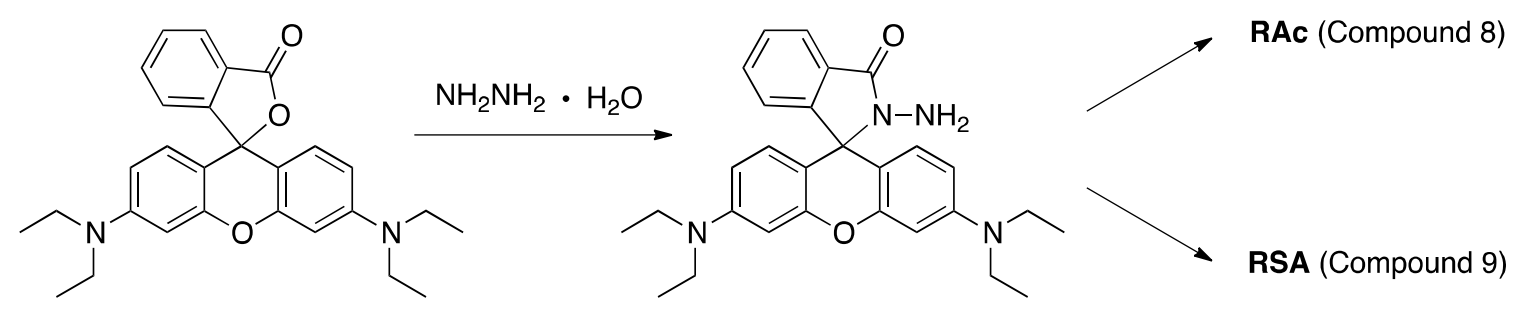

Scheme 10. Synthesis of Rhodamine derivatives.

The second step follows typical imine bond formation chemistry, which was previously discussed.

The reaction products have been characterized with UV-Vis, fluorescence, and ${ }^{1} \mathrm{H}$ NMR spectroscopy. The resulting compounds are nearly colorless and nonfluorescent in the absence of acid or metal ions.

\subsubsection{Spectroscopic Evaluation}

Fluorescence spectroscopy was employed to assess the sensitivity of the potential corrosion indicators and to compare their selectivity for aluminum ions. RAc was found to exhibit a 110-fold increase in emission intensity in the presence of one equivalent of aluminum nitrate. This fluorophore has been reported to be a competent indicator of $\mathrm{Fe}^{3+}$ ions in epoxy coatings. ${ }^{17}$ A comparison of the fluorescence spectra of RAc in the presence of equal amounts of various metal ions is shown in Figure 10. The fluorophore is most sensitive for $\mathrm{Al}^{3+}$ ions, but also shows a large increase in fluorescence intensity in the presence of $\mathrm{Fe}^{3+}$. A 3.5-fold disparity in emission intensity from $\mathrm{Fe}^{3+}$ to $\mathrm{Al}^{3+}$ was observed for this compound. 


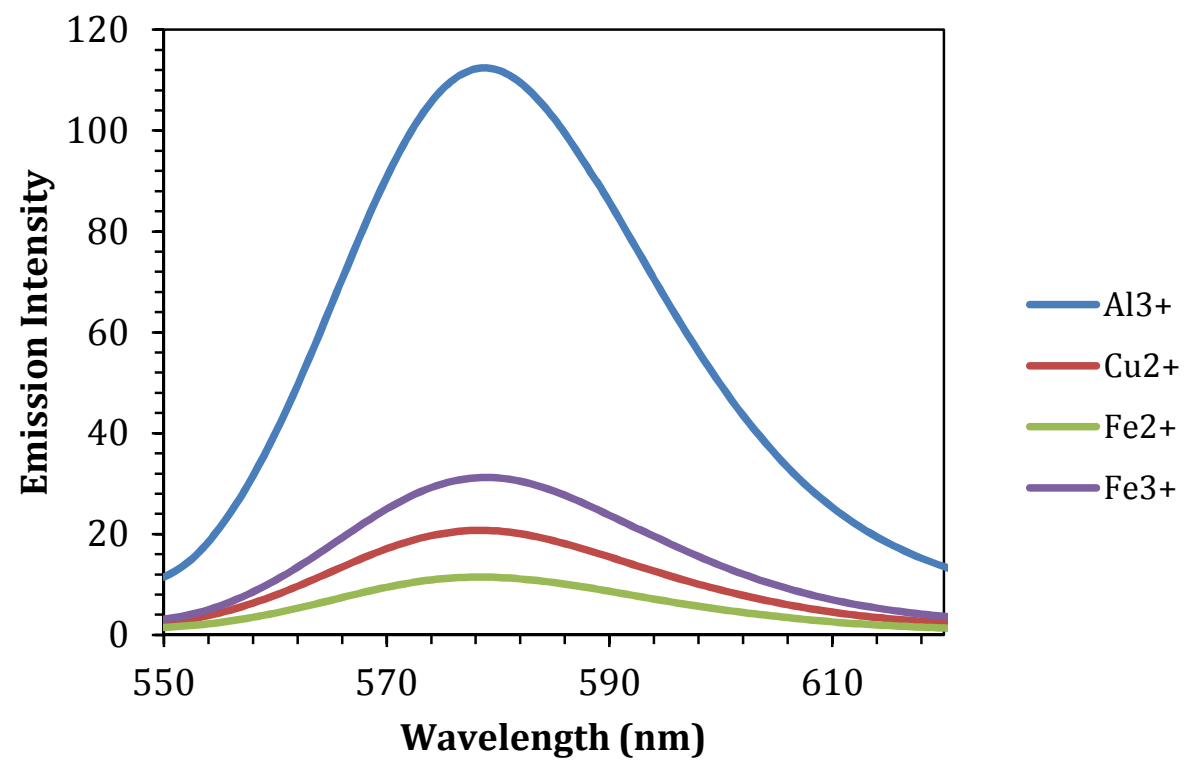

Figure 10. Fluorescence response of RAc in the presence of various metal ions.

The limited selectivity of RAc for aluminum ions proved to be a disadvantage for our proposed application. Our test panel alloy, Al-2024 T3, is composed primarily of aluminum but contains appreciable quantities of other alloying metals including copper and iron. The tendency of RAc to bind copper and iron ions, although slight, represents an interference in the detection of aluminum corrosion. Therefore, in an effort to improve the selectivity of the fluorescent indicator, RSA was synthesized. Unlike RAc, the binding site of RSA is rich in oxygen, similar to the Schiff base indicators that have been shown to bind aluminum ions (Figure 11). 


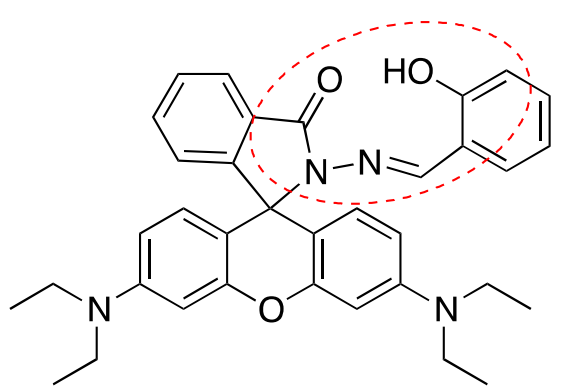

RSA (Compound 8)

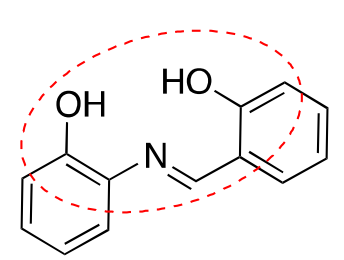

SOAP (Compound 5)

Figure 11. Similarity of the metal binding sites of RSA and SOAP.

As expected, the novel fluorophore, RSA, was found to have greater

selectivity for $\mathrm{Al}^{3+}$, as shown in Figure 12. Our studies suggest that this fluorophore is 10-fold more sensitive for $\mathrm{Al}^{3+}$ than $\mathrm{Fe}^{3+}$, the next highest signal. However, RSA is less sensitive, exhibiting only a 70-fold increase in fluorescence intensity following the addition of aluminum ions. This tradeoff lent importance to both Rhodamine compounds, thus both were appointed for further study.

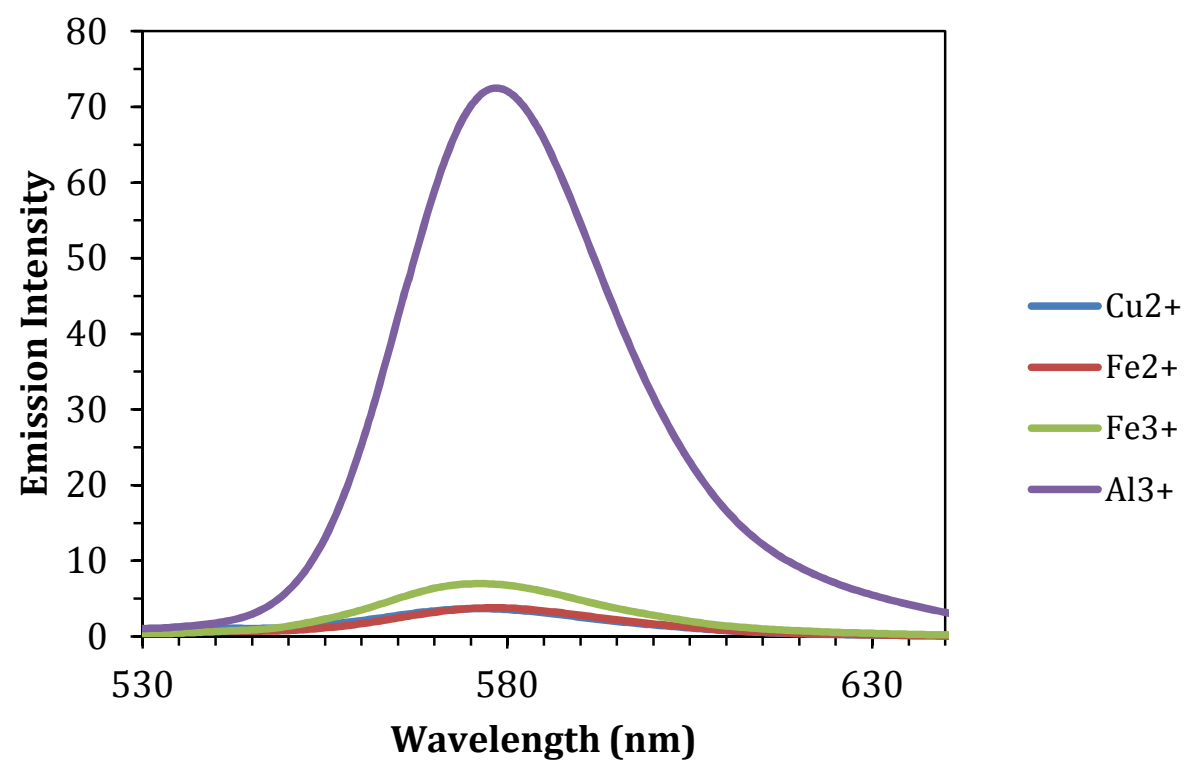

Figure 12. Fluorescence response of RSA in the presence of various metal ions. 


\subsubsection{Binding of Aluminum Ions by Rhodamine-based Fluorophores}

A recent publication suggested a method of fluorescence activation for RAc alternative to the binding of aluminum ions—an acid-promoted hydrolysis that results in a ring-opened, conjugated molecule that is fluorescent and incapable of binding aluminum ions. ${ }^{21}$

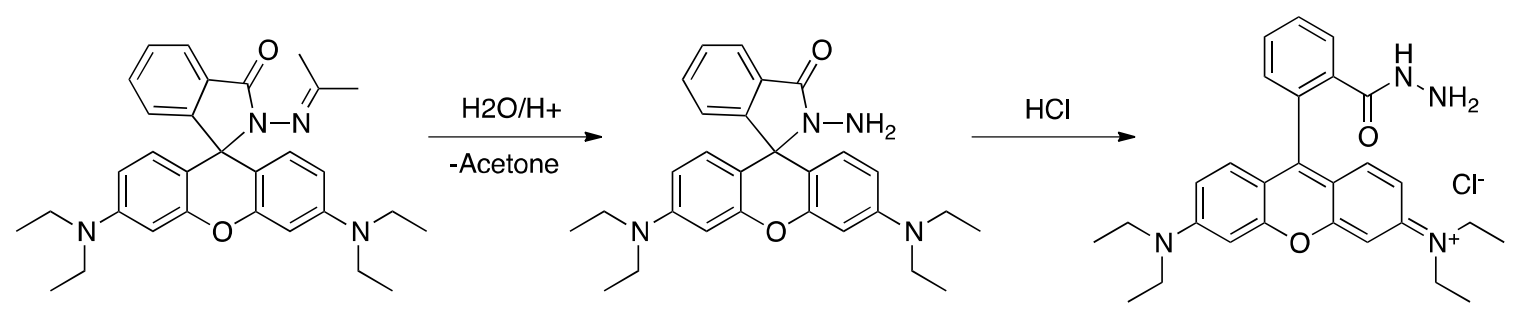

Scheme 11. Proposed mechanism of acid-promoted hydrolysis of RAc. ${ }^{21}$

Augustyniak and Ming found that fluorescence activation of RAc was induced via the addition of a small amount of $\mathrm{HCl}^{21}{ }^{21}$ Additionally, fluorescence titration revealed a linear relationship between fluorescence intensity and acid concentration, a behavior similar to the chelation-enhanced fluorescence observed in our lab for RAc and RSA in the presence of $\mathrm{Al}^{3+} .{ }^{21}$ In light of this information, it became necessary to invesitgate the chelating capability of these ligands. $\mathrm{Al}^{3+}$ is a strong Lewis acid. An aqueous $100 \mu \mathrm{M}$ solution of aluminum nitrate has a pH of $\sim 2$, owing to the ability of the $\mathrm{Al}^{3+}$ ion to hydrolyze water, resulting in the formation of acidic protons:

$$
\begin{gathered}
\mathrm{Al}^{3+}+\mathrm{H}_{2} \mathrm{O} \rightarrow \mathrm{Al}(\mathrm{OH})^{2+}+\mathrm{H}^{+} \\
\mathrm{Al}(\mathrm{OH})^{2+}+\mathrm{H}_{2} \mathrm{O} \rightarrow \mathrm{Al}(\mathrm{OH})_{2}^{+}+H^{+} \\
\mathrm{Al}(\mathrm{OH})_{2}^{+}+\mathrm{H}_{2} \mathrm{O} \rightarrow \mathrm{Al}(\mathrm{OH})_{3}+\mathrm{H}^{+}
\end{gathered}
$$

Aluminum nitrate solutions buffered with sodium acetate $(\mathrm{pH}=5.5)$ and triethylamine acetate $(\mathrm{pH}=7.0)$ were prepared to investigate the relationship between 
$\mathrm{pH}$ and fluorescence emission intensity. Addition of aluminum nitrate in triethylamine acetate buffer resulted in increased fluorescence from RSA, confirming that aluminum is capable of generating the fluorescent complex.

Like the findings of Augustyniak and Ming, fluorescence titration of a solution of RAc or RSA with $\mathrm{HCl}$ resulted in a linear increase in emission intensity dependent on the concentration of $\mathrm{HCl}$. This behavior, shown in Figure 13, is similar to that observed when titrating with aluminum nitrate.
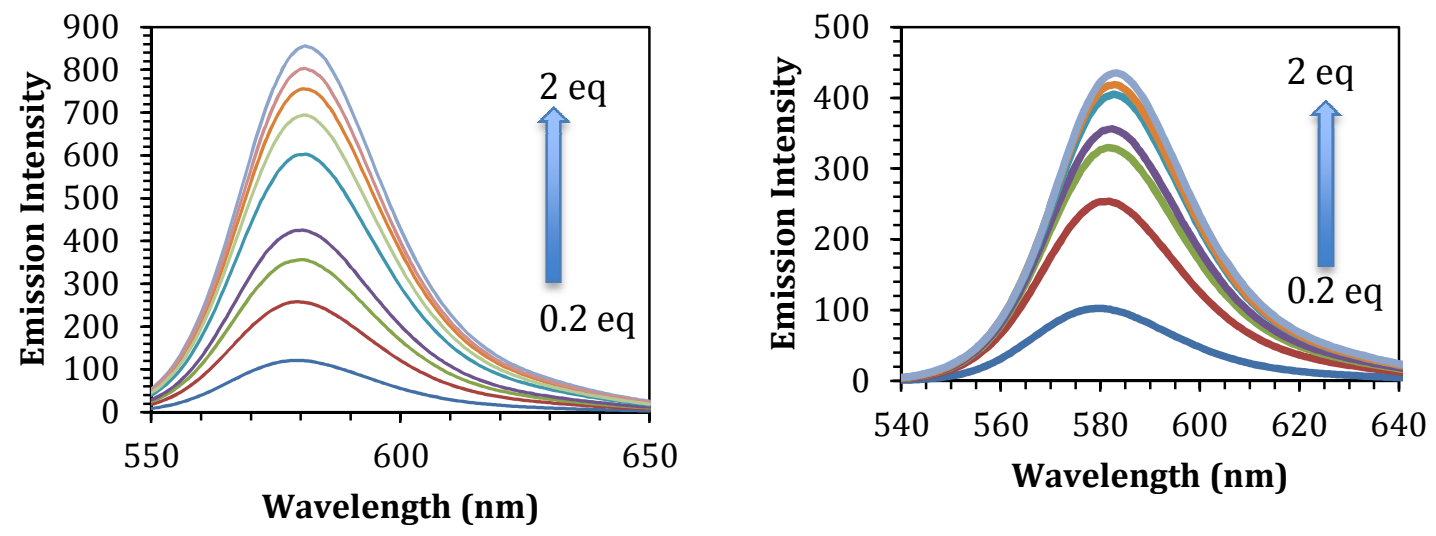

Figure 13. Side by side comparison of titration of a solution of RSA in acetonitrile with $\mathrm{HCl}$ (left) and aluminum nitrate (right).

While similar in appearance, the rate at which the emission intensity increases with increasing amounts of $\mathrm{HCl}$ is different than when titrating with aluminum nitrate. Additionally, the emission intensity of the aluminum titration reaches a maximum at $\sim 0.5$ eqivs. of aluminum due to the incorporation of all free aluminum ions into the RSA-Al complex. A maximum intensity is also observed for the $\mathrm{HCl}$ titration. However, the maximum intensity value occurs at one equivalent of $\mathrm{H}^{+}$to RSA, reaching this point once each individual RSA molecule has undergone fluorescence activation. The most probable explanation of the decrease in intensity observed after this point is the hydrolysis of the ligand's imine bond resulting in the 
formation of rhodamine-hydrazine. To further illustrate this behavior, plots of emission intensity versus equivalents of titrant are provided for both experiments in Figure 14.
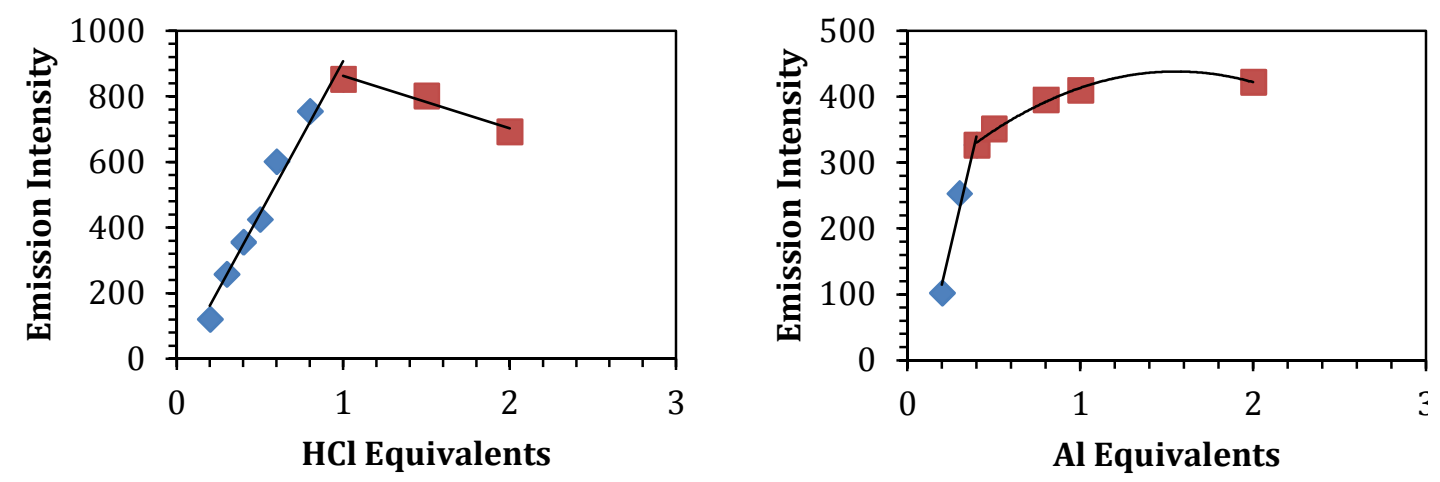

Figure 14. Plots of emission intensity as a function of equivalents of titrant for $\mathrm{HCl}$ and aluminum nitrate fluorescence titration experiments.

${ }^{1} \mathrm{H}$ nuclear magnetic resonance spectroscopy was employed to further investigate the mechanism responsible for fluorescence in this compound. A typical ${ }^{1} \mathrm{H}$ NMR spectrum of RSA in deuterated acetonitrile is shown in Figure 13. Proton nuclear magnetic resonance analysis showed a triplet at $1.14 \mathrm{ppm}$ and a quartet at $3.38 \mathrm{ppm}$, corresponding to the $\mathrm{CH}_{3}$ and $\mathrm{CH}_{2}$ protons respectively of the compound's two tertiary amines on the symmetrical region of the structure. Further downfield, a series of aromatic peaks appear in the range 6.38-7.96 ppm. 


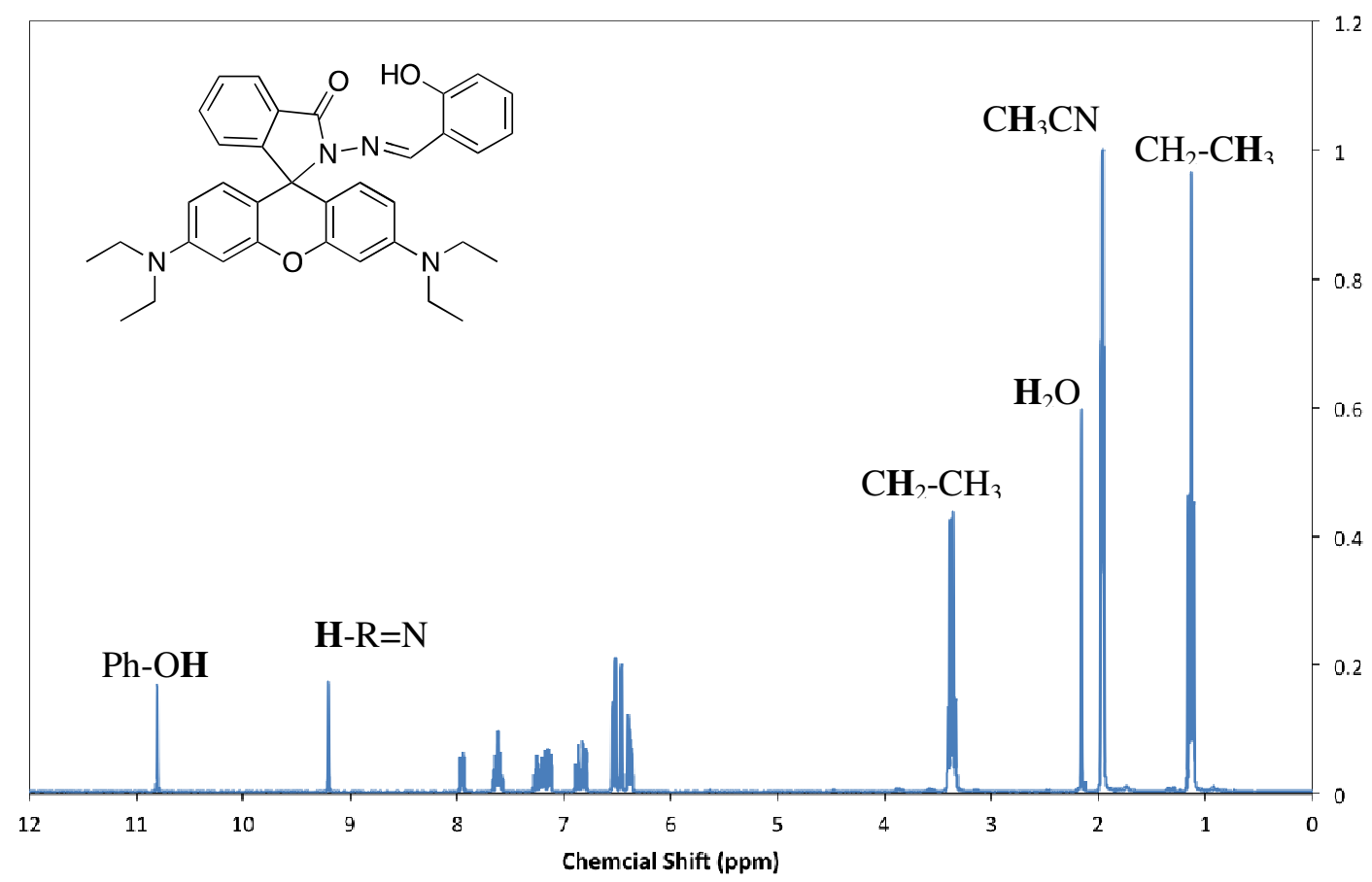

Figure 15. ${ }^{1} \mathrm{H}$ NMR spectrum of RSA in acetonitrile-d3.

A singlet at $9.20 \mathrm{ppm}$ coincides with the proton adjacent to the ligand's imine bond. The peak furthest downfield, located at $10.8 \mathrm{ppm}$, is the phenolic peak of the salicylaldehyde moiety. When $\mathrm{HCl}$ in deuterated water is added to the RSA solution, a change in the quantity, splitting, and location of the peaks in the aromatic region was observed (Figure 14). Additionally, following $\mathrm{HCl}$ addition, the phenolic peak disappeared completely-most likely the result of proton exchange between the phenolic proton and the deuterium of $\mathrm{D}_{2} \mathrm{O}$. 


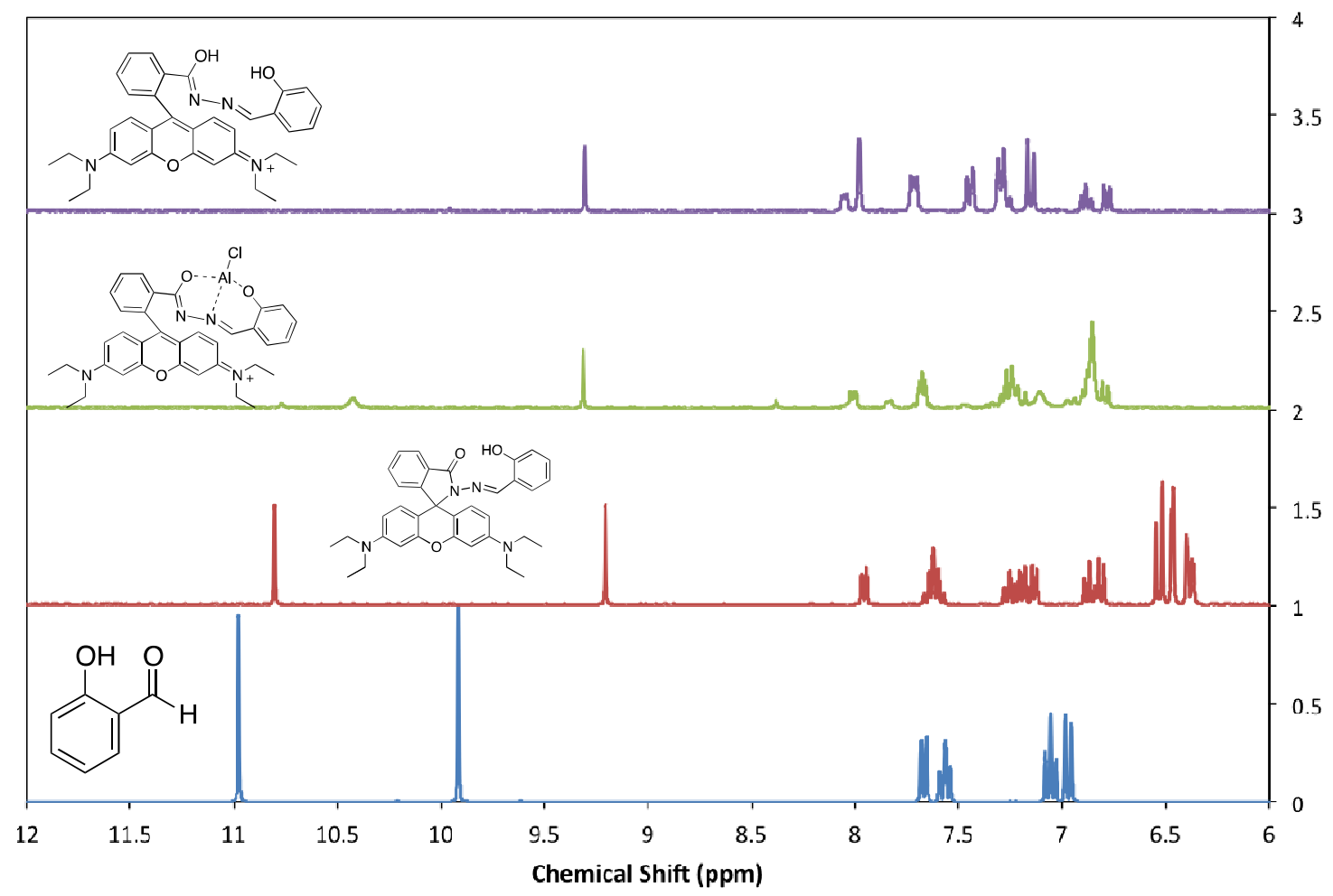

Figure 16. ${ }^{1} \mathrm{H}$ NMR evaluation of RSA and its starting materials. Spectra are, from bottom to top: salicylaldehyde, rhodamine-salicylaldehyde (RSA), RSA and Al, RSA and $\mathrm{DCl}$. All experiments were conducted in acetonitrile-d3.

The product of the reaction between $\mathbf{R S A}$ and $\mathrm{HCl}$ is fluorescent. In addition, ${ }^{1}$ H NMR spectra do not show formation of free salicyladehyde, as evident by the absence of the aldehyde peak at $\sim 10 \mathrm{ppm}$, suggesting that hydrolysis of the imine bond in RSA is not occurring. Addition of one equivalent of aluminum nitrate results in a distinctive spectrum that more closely resembles the spectrum of the unreacted RSA. The difference in spectra observed when adding DCl versus aluminum implies the formation of two unique chemical species. Although acid hydrolysis can generate a fluorescent state in RSA, it is also apparent that aluminum ions can bind to the fluorophore and generate a fluorescent complex. It is likely a combination of these two mechanisms that gives rise to emission by RSA in epoxy coatings in the presence of aluminum ions. 
The ${ }^{1} \mathrm{H}$ NMR spectra suggest that the binding of aluminum leads to the observed fluorescence activation of the indicator. However, they do not rule out the possibility of an acid-promoted activation mechanism. The binding interaction between aluminum ions and RSA was studied with ${ }^{27}$ Al NMR spectroscopy. By monitoring the signals associated with aluminum species in solution, I believe I have proven definitively that the fluorophore is capable of binding aluminum ions in solution.

${ }^{27} \mathrm{Al}$ NMR spectra of aluminum nitrate in a 50:50 mixture of toluene/methanol- $\mathrm{d}_{4}$ showed two regions of import: a broad peak downfield corresponding to a number of overlapping coordinated aluminum species, and a series of narrower peaks upfield for free, coordinated, and hydrated aluminum ions (Figure 17). Titration of the aluminum solution with RSA caused the integration value of the "free aluminum" ion peak to decrease. Indeed, this free aluminum peak approaches the shape and integration of the external standard $\left(\mathrm{Al}^{3+}\right.$ in deuterium oxide $)$ as more fluorophore is added. The decrease of the "size" of this peak, along with the visible change in color and increased fluorescence observed upon addition of the indicator, confirms the binding of $\mathrm{Al}^{3+}$ ions. 


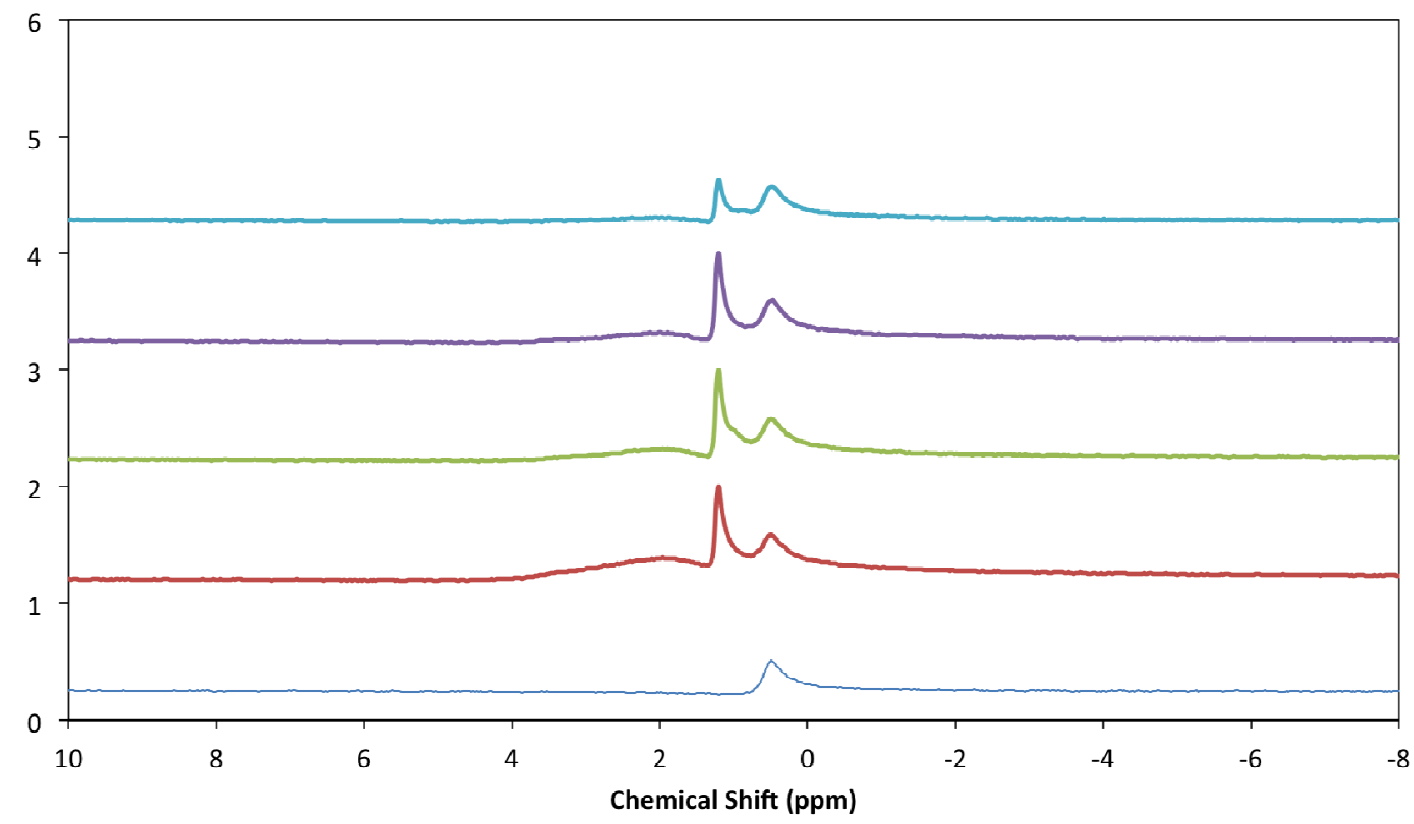

Figure 17. Spectra are, from bottom to top: aluminum nitrate in deuterium oxide (external standard), $\mathrm{Al}^{3+}$ in 50:50 toluene/methanol-d4; a 3:2 excess of aluminum ions to RSA in the same solvent; a stoichiometric amount of RSA; a 3:2 excess of RSA.

An RSA-Al complex was synthesized from RSA and diethyl aluminum chloride in toluene, shown in Scheme 10. The resulting compound was insoluble in toluene and was isolated by vacuum filtration. Mass spectrometry of the violet RSA$\mathrm{Al}$ crystals showed three important $\mathrm{M}^{+}$peaks at 561,622 , and 1146 , correlated to RSA, the RSA-AlCl complex, and a bis-coordinated RSA-Al complex, respectively (Figure 18). 


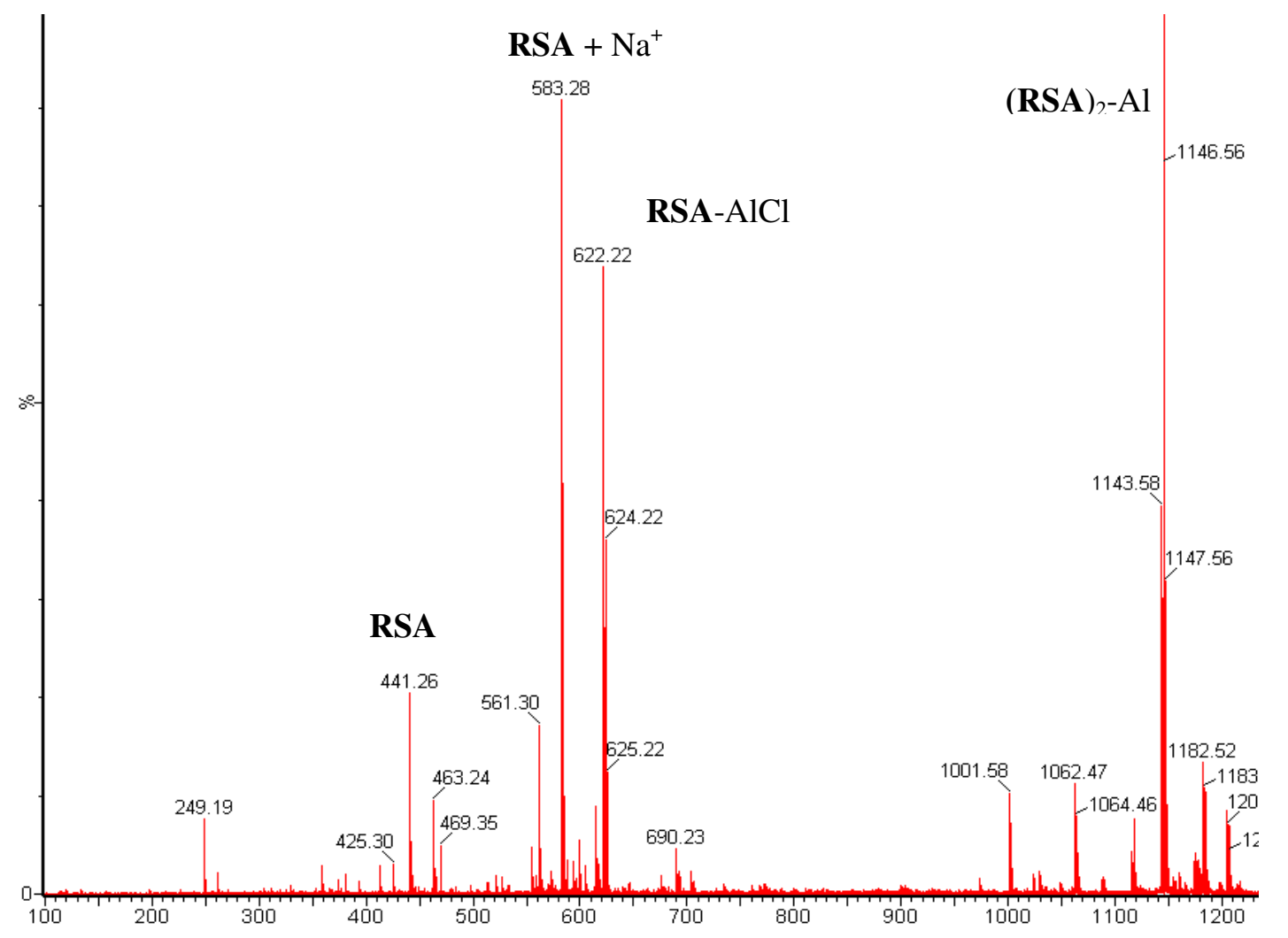

Figure 18. Mass spectrum of the RSA-Al complex.

The presence of the aforementioned species in the mass spectrum serves as further evidence of the indicator's ability to bind aluminum ions, and suggests that the binding equilibrium between RSA and aluminum may not be stoichiometric. Monoand bis-coordinated complexes are possible, and will factor into the binding equilibrium between aluminum and RSA.

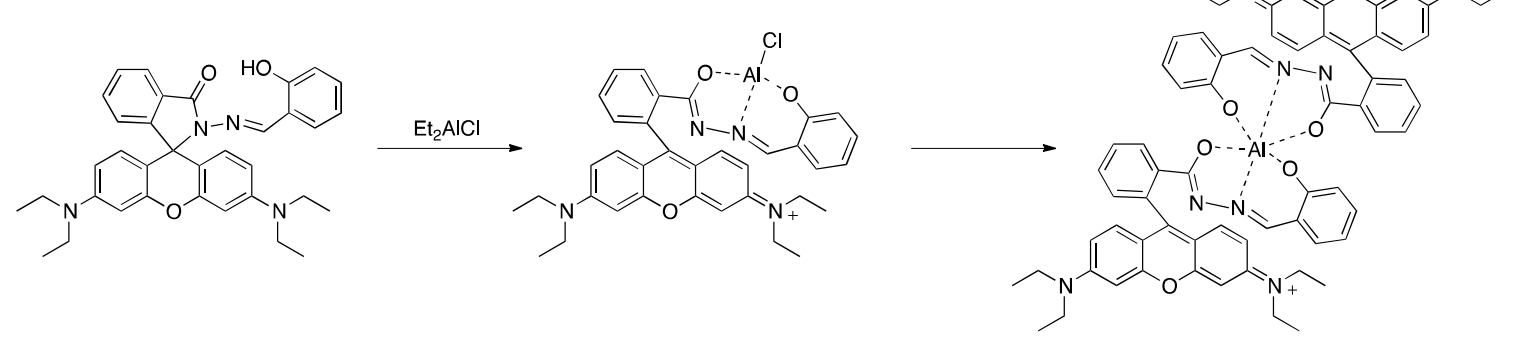

Scheme 12. Formation of mono- and bis-coordinated aluminum complexes from diethyl aluminum chloride. 
Investigation of the binding stoichiometry by absorption spectroscopy of RSA in acetonitrile (Figure 19) suggests that a binding stoichiometry of 2:1 RSA/Al is preferred.

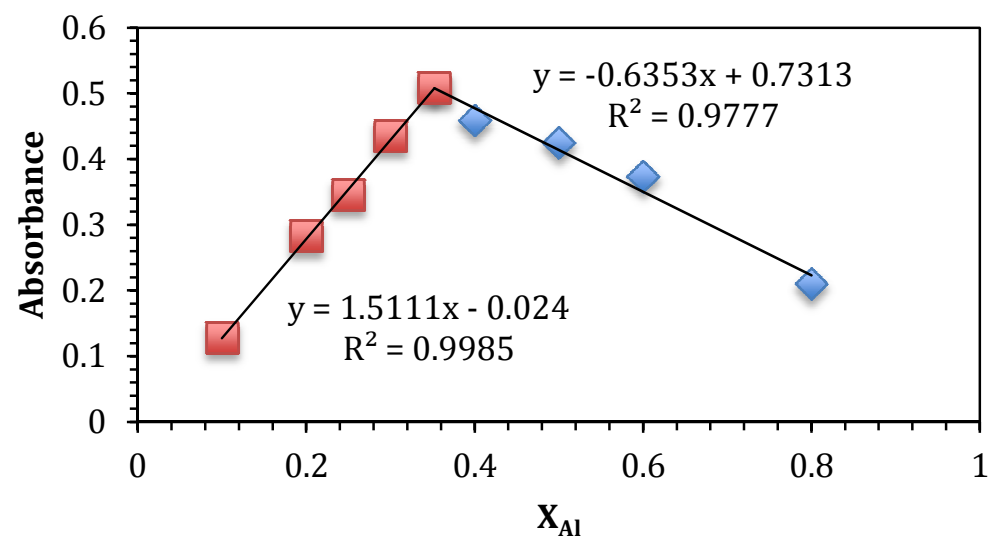

Figure 19. Job plot of RSA with $\mathrm{Al}^{3+}$ in acetonitrile; $\mathrm{Y}$-intercept $=0.352$.

\subsubsection{RSA in Epoxy Films}

The dispersibility of RSA in a solvent-borne epoxy coating was evaluated by dissolving the indicator in a small amount of toluene and then mixing the organic solution into the epoxy formulation prior to application. The epoxy coating containing the indicator was spread on glass slides and allowed to cure for 24 hours at $70^{\circ} \mathrm{C}$. Optical microscopy revealed no aggregation of the dye. The Typhoon imager was employed to monitor the emission intensity of the slides after submerging into a 0.5 $\mathrm{M}$ aqueous solution of aluminum nitrate. The fluorophore-containing epoxy coatings showed a small background emission. Soaking of the glass slides in aluminum nitrate solution for 1 hour resulted in an increase of $48 \%$ in the emission intensity of the epoxy films. Fluorescence analysis of the soaking solutions showed no fluorescence, suggesting that the indicator does not leech from the film. 

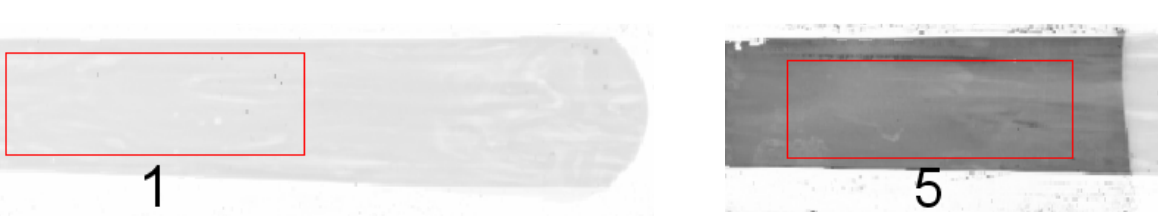

Figure 20. Imaging with the Typhoon Trio+ revealed an increase in fluorescence emission intensity after soaking for one hour in aluminum nitrate solution.

To obtain a calibration plot, slides coated with epoxy that contained RSA were submerged into solutions of increasing aluminum nitrate concentration. However, calibration by absorption proved difficult, with no clear linear relationship emerging between emission intensity and the concentration of the aluminum nitrate solution in which the slide was soaked. Calibration with respect to aluminum concentration was also hindered by the inability to add aqueous aluminum ions to the solvent-borne epoxy formulation. To obtain calibration data, known amounts of the RSA-Al complex were added to epoxy coatings on glass slides. Unfortunately, the charged complex was insoluble in the solvent-based epoxy formulation and aggregated once its dispersing solvent (ethanol) had evaporated. An anionic surfactant, Triton X-200K, was added to the epoxy formulation to improve the dispersion of the aluminum complex in the dry film. This surfactant, composed of both greasy hydrophobic and charged regions, associated with the cationic complex, stabilizing the charge in the dry film. 


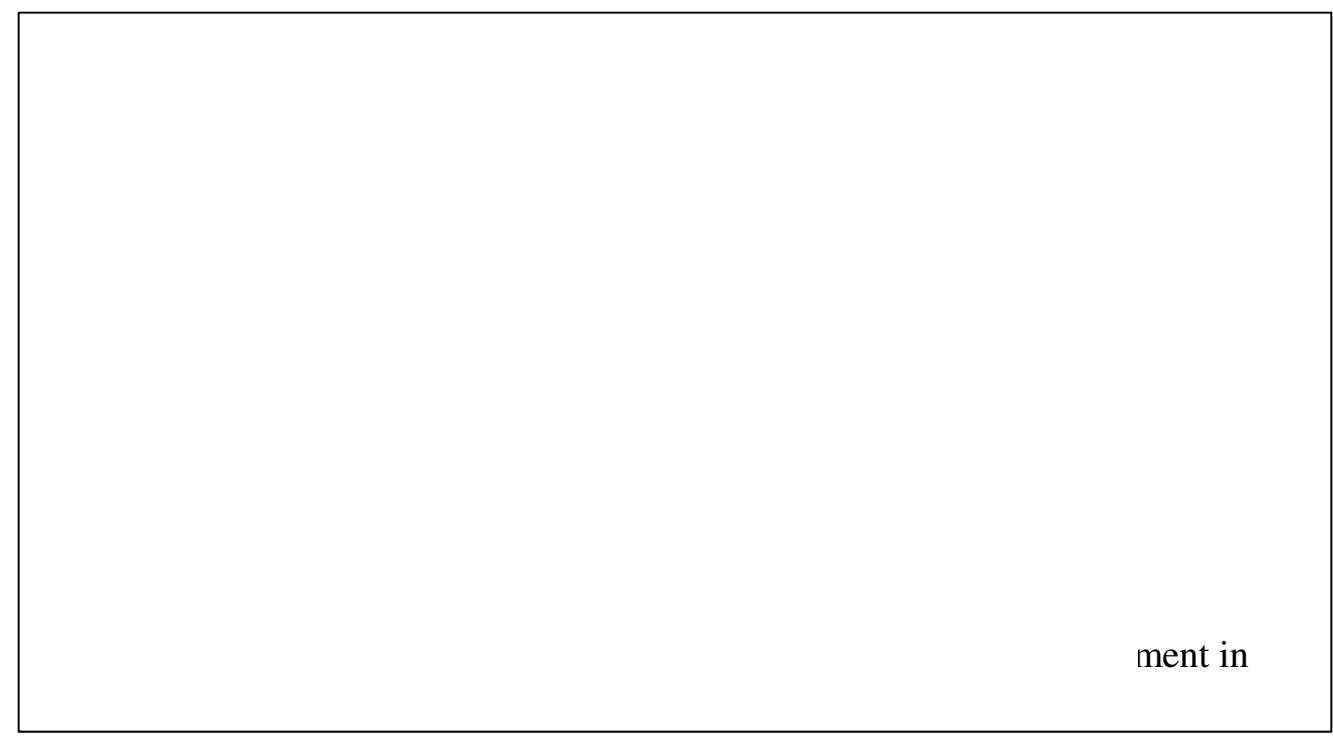

Epoxy coatings containing increasing amounts of the $\mathbf{R S A}-\mathrm{AlCl}$ compound show a linear increase in emission on the Typhoon imager, as shown in Figure 22. In epoxy coating samples, the total quantity of RSA is fixed at $1 \mathrm{wt} \%$ so that background emission of the fluorophore can be considered. The average emission intensity over a predetermined area of the coating is used in the calibration plot.
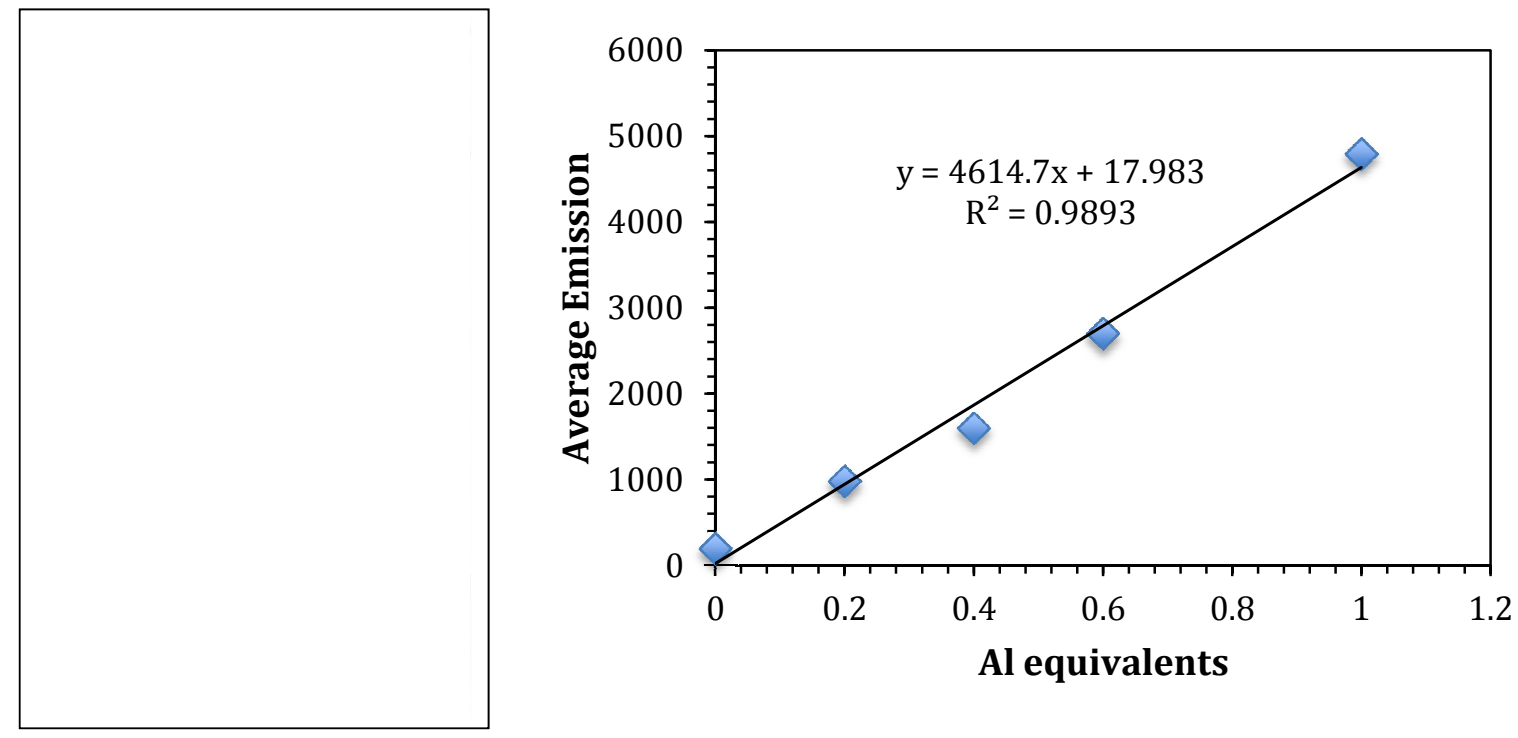

Figure 22. Calibration of the RSA-Al complex in epoxy coatings. Shown are the fluorescence image (left) and the accompanying calibration plot (right). 


\subsubsection{Immersion Corrosion Studies}

The ability of RSA to detect corrosion on aluminum substrates, and further to quantify the degree of corrosion relative to other samples, was evaluated by coating Al-2024 T3 aluminum coupons with an epoxy coating containing 1\% w/w of the fluorescent indicator and submerging them into a $3 \% \mathrm{w} / \mathrm{v}$ solution of $\mathrm{NaCl}$. The emission intensity of the films was monitored daily using the Typhoon confocal imager. Over the course of the experiment, the development of pits in the aluminum substrate and blisters in the epoxy film were observed, with their diameter increasing over time (Figure 23).

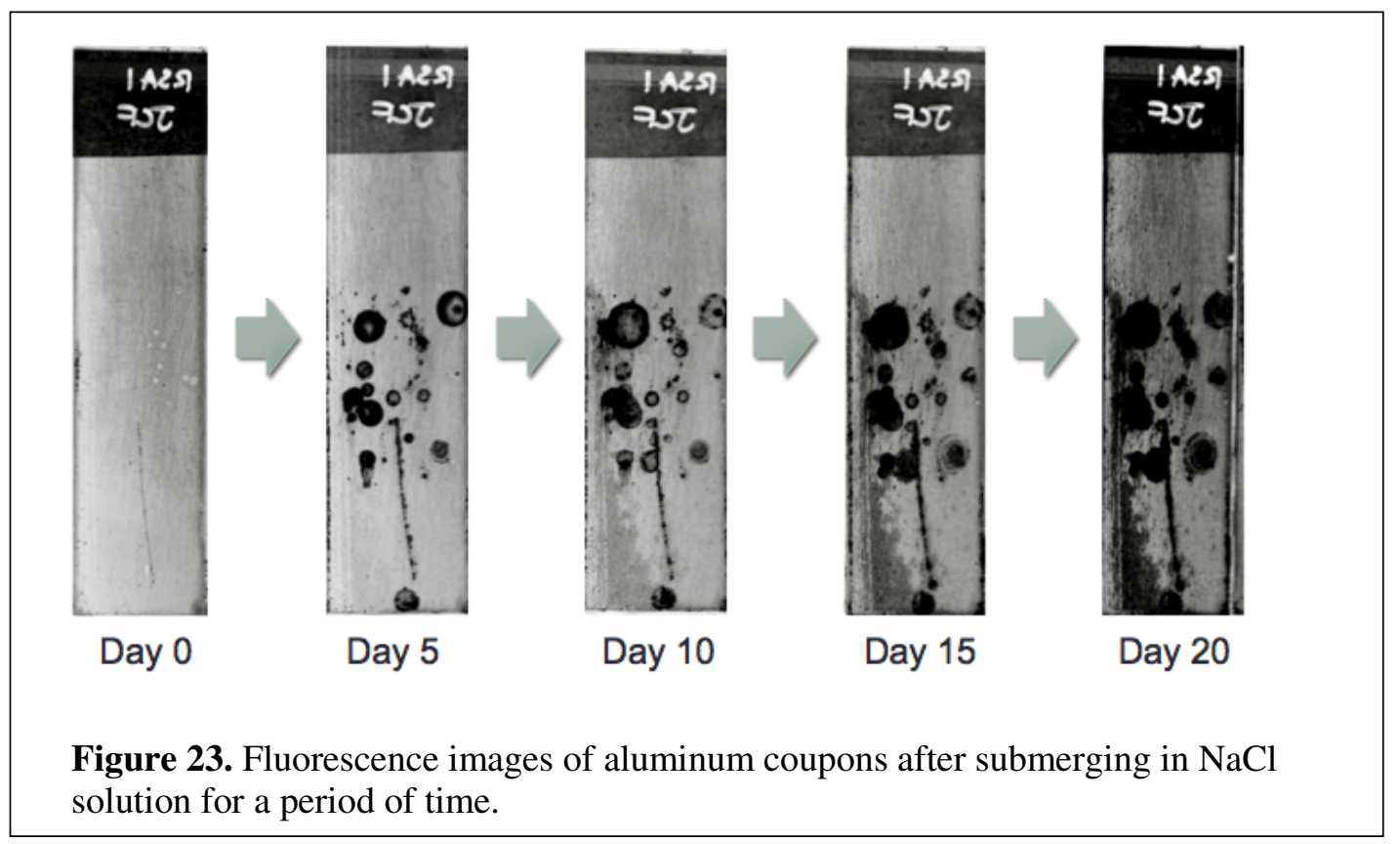

To track, quantitatively, the increase in corroded area, a plot of average emission intensity as a function of time was constructed. This plot is shown in Figure 24. Unfortunately, the resulting relationship deviated greatly from linearity. Therefore, it became imperative to investigate a number of variables that might have interfered with the test results. For example, substantial volumes of water were observed (via optical microscopy) to exist beneath the epoxy film. This trapped water 
was found to have a detrimental effect on the measurement of fluorescence intensity - the aqueous hemispheres scattered and absorbed light on its way to the substrate and back to the detector. Other experimental variables included the cleanliness of the Typhoon imager's glass surface, the presence/absence of precipitated salt on the surface of the epoxy films, temperature, and the time interval between removing the coupons from the soaking solution and scanning them with the Typhoon imager. To eliminate these potential sources of experimental error, a new procedure was developed which involved: (1) drying the samples for 1 hour at $70^{\circ} \mathrm{C}$ prior to imaging, (2) cleaning the glass surface of the Typhoon imager with deionized water, and (3) rinsing of the epoxy films to remove any precipitated salt. The immersion corrosion test was repeated using this protocol. In addition, a new method of evaluating the data was employed which involved the determination of the area of pixels with an intensity value greater than a specified threshold as opposed to the average emission intensity over a given area. This was accomplished using the software by using the "spot finder" utility, which calculated the number of pixels on the fluorescence image that had emission intensities greater than a specified threshold. Corroded area was plotted against the time of immersion. Although imperfect, a clear trend emerges from the data, showing an increase in the corroded area over time. 


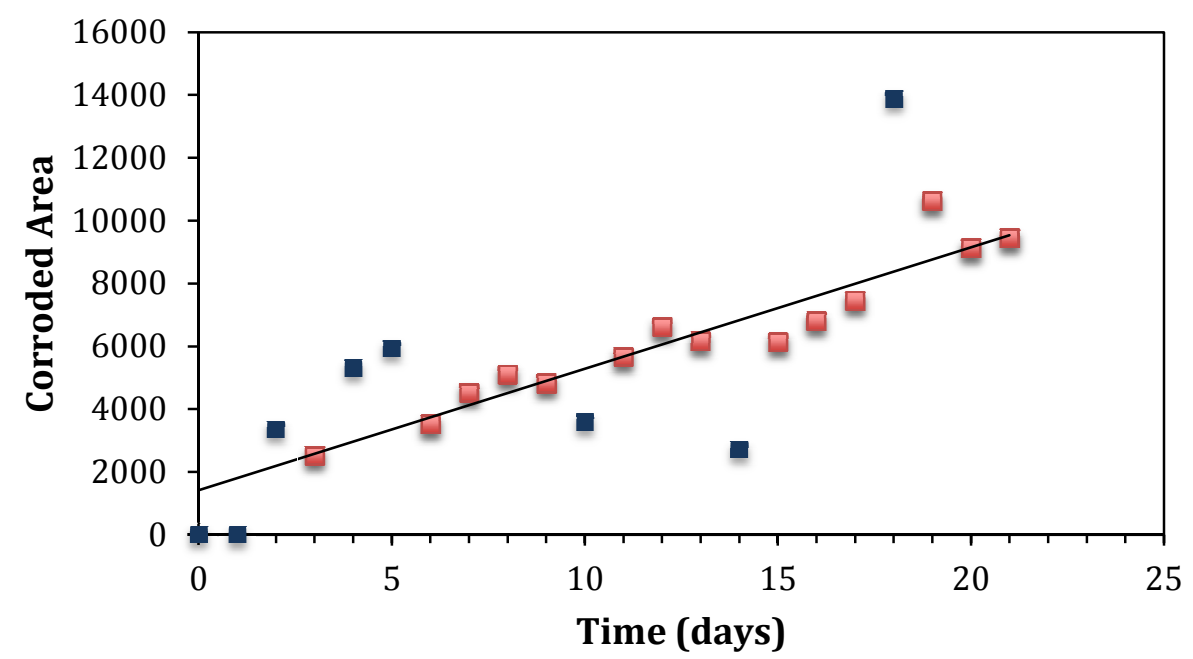

Figure 24. Plot of corroded area as a function of time. Data points that differ more than $30 \%$ from the linear trend are colored blue.

\subsubsection{Salt Spray Corrosion Studies}

The salt spray test is a standardized method to evaluate the corrosion resistance of coated metals. The corrosion of samples is accelerated via exposure to a concentrated salt solution in a corrosive environment. Al-2024 T3 aluminum panels were coated with the solvent-borne epoxy containing $1 \%$ w/w (dry) RSA. The Q-Fog instrument was configured to run the prohesion test (ASTM G85), and the coated test panels were imaged daily over a period of $360 \mathrm{hr}$ with the Typhoon confocal imager. Like the immersion test, the salt spray test suffered from experimental error stemming from a variety of experimental variables. In addition, the salt fog chamber malfunctioned at multiple instances throughout the duration of the test, further complicating the results. Still, a linear trend was observed in the data. Images taken at intervals of five days are shown in Figure 25. 


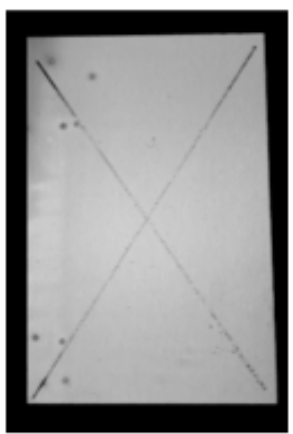

Day 0

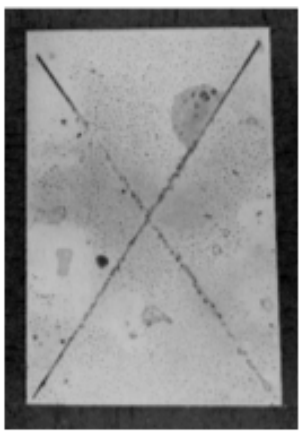

Day 5

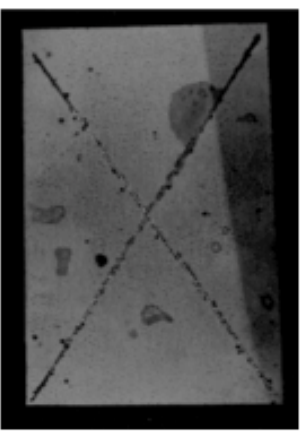

Day 10

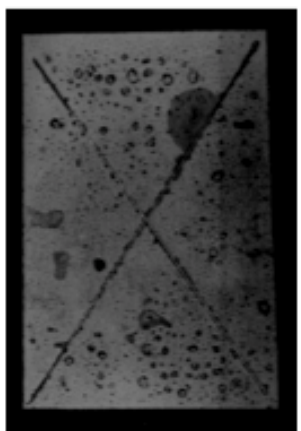

Day 15

Figure 25. Fluorescence images of aluminum panels after salt fog exposure.

It is clear that the extent of corrosion increased throughout the lifetime of the experiment. Analysis of the fluorescent images with the ImageQuant software revealed a linear trend in the data. Unfortunately, the method of analysis utilized for the immersion testing could not be applied - the computer on which the analysis was conducted lacked the physical memory to perform the "spot finder" operation. Instead, the average emission intensity measured over a given area was plotted against time (Figure 26). Given that the emission intensity is proportional to the quantity of activated fluorophore, which is itself proportional to the amount of $\mathrm{Al}^{3+}$ ions produced by the corrosion process, the average emission intensity is a direct measure of the extent of corrosion. 


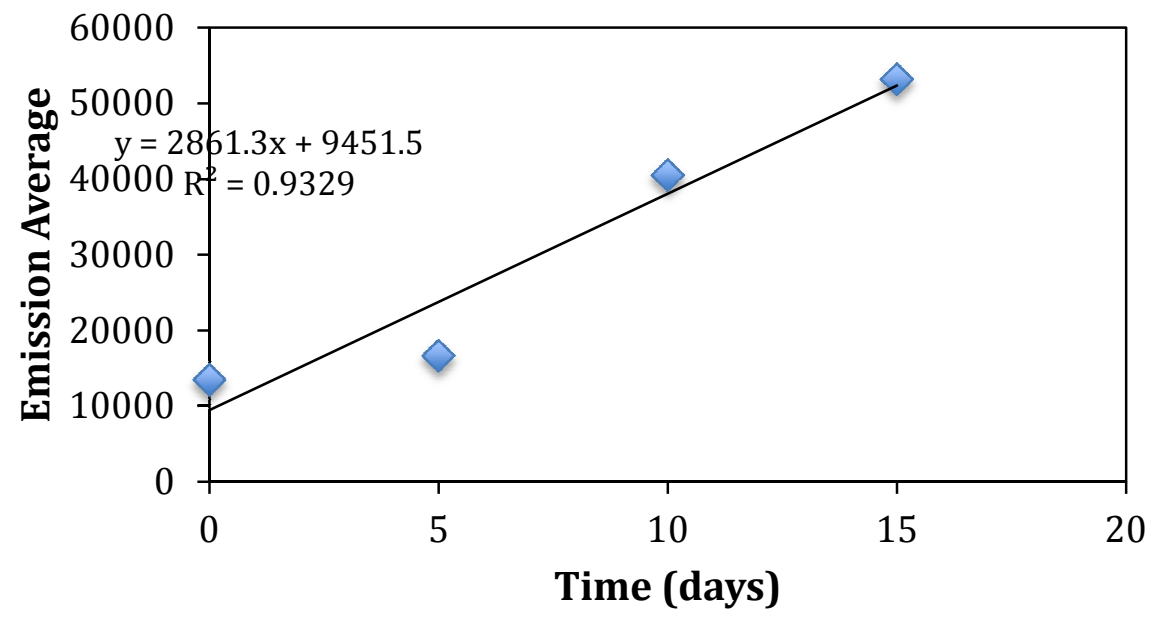

Figure 26. Plot of average emission intensity over time.

\subsubsection{Spray-on Application of RSA}

"Spray tests" were conducted to investigate a potential application of the fluorophore, in this case, to detect corrosion that has already occurred. To this end, aluminum panels that had been corroded for 3,000 hours in the salt fog chamber were sprayed with a $1 \% \mathrm{w} / \mathrm{v}$ RSA solution in toluene. The panels were imaged before and after spraying with the Typhoon imager (Figure 27).

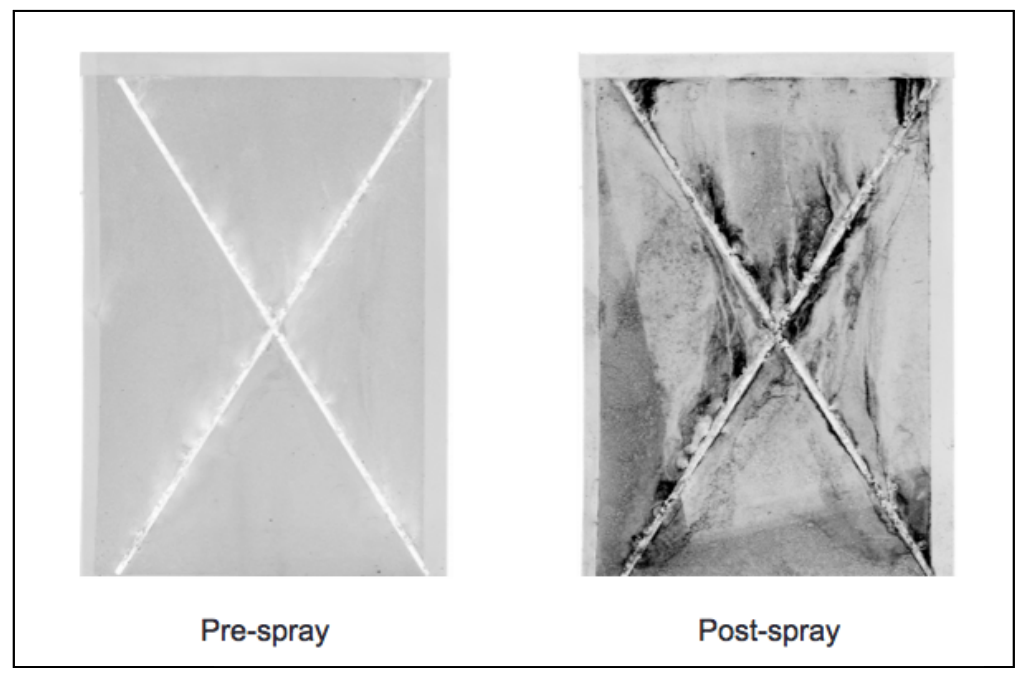

Figure 27. Fluorescence images of corroded aluminum panels sprayed with a $1 \% \mathrm{w} / \mathrm{v}$ solution of RSA. 
A vast increase in fluorescence emission was observed following spray application. The chelation reaction occurred rapidly. The surface of the panel became pink almost instantly when the RSA solution was applied to the surface, and the panel fluoresced bright orange under UV-light. 


\section{Chapter 5: Conclusions}

Eight fluorophores were evaluated as indicators of corrosion for coated aluminum. Rhodamine-salicylaldehyde was selected as a candidate for further study based on (1) its ease of synthesis, (2) the long-term stability of the fluorophore in solution, (3) the selectivity of the fluorophore for aluminum, (4) excitation and emission wavelengths within the operational range of our Typhoon imager and (5) the solubility of the fluorophore in organic solvents and its related dispersability in the solvent-based epoxy formulation. A recent publication suggested an alternative method of fluorescence activation, thus it became necessary to confirm the ability of the fluorophore to bind aluminum ions. Following thorough ${ }^{1} \mathrm{H}$ and ${ }^{27} \mathrm{Al}$ NMR spectroscopy, mass spectrometry, and fluorescence analysis, the ability of RSA to bind aluminum ions was confirmed.

The corrosion of aluminum panels coated with epoxy was investigated. In general, the emission intensity of coated aluminum panels in a corrosive environment, as measured by the Typhoon imager, increased over time. The optimal loading of RSA was determined through the process of aluminum panel corrosion. Fluorescent regions were observed to appear as early as three days into the corrosion process. These fluorescent areas, corresponding to corroding regions, increased in size with time. Once experimental variables had been eliminated, a linear relationship between corroded area and corrosion time was observed. 


\section{Chapter 6: Potential Applications}

Rhodamine-salicylaldehyde has been demonstrated to be a proficient, quantitative indicator of corrosion. Using the ImageQuant software, two methods of analysis can be performed - a calculation of the average emission intensity over a given area, and the determination of the area of pixels in the scanning region that have an emission intensity value greater than a specified threshold. Time course measurements have proven to be useful for tracking the progress of corrosion, although these required vigorous optimization. The spray-on method, however, proved to be a powerful technique to compare, qualitatively, the extent of corrosion at a given instance.

Dispersing RSA in primers and coatings applied to industrial products may not be feasible. To achieve the levels of indicator employed in our experiments on the large scale, gram scale, hundred gram scale, and even kilogram scale masses could be required. It may be that the synthesis of RSA can be scaled to achieve these quantities. However, further investigation of the interaction between the fluorophore and other formulation components, or of the ability of the emission of RSA to be detected in applications where a topcoat is used, is needed before this route is pursued.

It is more likely that RSA will prove useful in the laboratory setting. Incorporation of the indicator into organic coatings on aluminum allows for the study of the corrosion of the underlying alloy. Provided that RSA does not interact with potential corrosion inhibitors, it can be used to evaluate their efficacy by comparing the quantitative "corrosion parameters" obtained from the ImageQuant software. However, as discussed previously, the quantitation of RSA emission in polymer films 
suffers from a high degree of variability, stemming from experimental variability such as the amount of water absorbed by the film, the film thickness, the presence of organic or inorganic residues (i.e. $\mathrm{NaCl}$ ) on the film surface, etc.

In contrast, the spray-on method endures none of these difficulties. Based on experience gleaned from experimental work, this method seems the most powerful, practical, and robust. The indicator, dissolved in an organic solvent, can be applied to test panels following corrosion. Using the ImageQuant software, the extent of corrosion, and by extension the efficacy of various corrosion inhibitors, can be quantified and compared. 


\section{References}

1. Koch, G. H.; Brongers, M. P. H.; Thompson, N.G.; Virmani, Y. P.; Payer, J.H. Corrosion Costs and Preventative Strategies in the United States. National Association of Corrosion Engineers 2002.

2. Chattopadhyay, A. K.; Zentner, M. R. Aerospace and Aircraft Coatings; Federation of Societies for Coatings Technology: Philadelphia, 1990; pp 1017.

3. Hinton, B. R. W.; In Metal Finishing, 1989.

4. Polmear, I. J. Light Alloys Metallurgy of the Light Metals; Arnold: London, 1995.

5. Muster, T. H.; Hughes, A. E.; Thompson, G. E. In Corrosion Research Trends; I. S. Wang Ed.; Nova Science Publishers, Inc., 2007.

6. Birbilis, N; Buchheit, R. G. Journal of the Electrochemical Society 2005, 152, B140.

7. Corrosion of Titanium and Titanium Alloys; ASM Handbook, Vol. 13; p. 675.

8. Wicks, Z. W. Corrosion Protection by Coatings; Federation of Societies for Coatings Technology: Philadelphia, 1987; p 11-13.

9. Greenfield, B. D. The use of fluorescent probes for the detection of under-film corrosion. Progress in Organic Coatings 2006, 57, 416-420.

10. Solomons, G.; Organic Chemistry, $4^{\text {th }}$ ed.; John Wiley \& Sons: New York, 1988.

11. White, C. E.; Lowe, C. S. Determination of Aluminum by Photometric Fluorescence Measurement. Ind. and Eng. Chem. 1940, 12, 229-231.

12. Gündüz, S. B.; Küçükkolbası, S.; Atakol, O.; Kılıç, E. Spectrofluorimetric determination of trace aluminum in diluted hemodialysis solutions.

Spectrochimica Acta Part A: Molecular and Biomolecular Spectroscopy 2005, 61, 913-921.

13. Jiang, C.; Tang, B.; Wang, R.; Yen, J. Spectrofluorimetric determination of trace amounts of aluminium with 5-bromo-salicylaldehyde salicyloylhydrazone. Talanta 1997, 44, 197-202.

14. Sibi, M. P.; Zong, Z. Determination of corrosion on aluminum alloy under protective coatings using fluorescent probes. Progress in Organic Coatings 2003, 47, 8-15.

15. Chambers, B.; Taylor, S. The high throughput assessment of aluminium alloy corrosion using fluorometric methods. Part I-Development of a fluorometric method to quantify aluminium ion concentration. Corrosion Science 2007, 49, 1584-1596.

16. Chambers, B.; Taylor, S. The high throughput assessment of aluminium alloy corrosion using fluorometric methods. Part II-A combinatorial study of 
corrosion inhibitors and synergistic combinations. Corrosion Science 2007, 49, 1597-1609.

17. Augustyniak, A.; Tsavalas, J.; Ming, W. Early Detection of Steel Corrosion via "Turn-On" Fluorescence in Smart Epoxy Coatings. ACS Appl. Mater. Interfaces 2009, 1, 2618-2623.

18. Zhang, X.; Shiraishi, Y.; Hirai, T. A new rhodamine-based fluorescent chemosensor for transition metal cations synthesized by one-step facile condensation. Tetrahedron Letters 2007, 48, 5455-5459.

19. Aoki, I.; Matsunaga, T.; Watanabe, K. Substituent-group effects on the fluorescence of aluminum-schiff base complexes and their application to the determination of aluminum. Bulletin of the Chemical Society of Japan 1989, 62, 2369-2374.

20. Kuzuya, M.; Usui, T.; Ito, S.; Miyake, F.; Nozawa, S.; Okuda, T. Substituent Effects and Structural Limitations in the Conversion of 3,3-Diaryl-phthalides to 4,4-Diaryl-3,4-dihydro-1(2H)-phthalazinones. Chem. Pharm. Bull. 1980, 28, 3561-3569.

21. Augustyniak, A.; Ming, W. Early detection of aluminum corrosion via 'turnon' fluorescence in smart coatings. Progress in Organic Coatings 2011, 71, 406-412.

22. Mansfield, F. Use of electrochemical impedance spectroscopy for the study of corrosion protection by polymer coatings. J. App. Electrochemistry 1994, 25, $187-202$.

23. Scho"nberger, A.; Virtanen, S.; Giese, V.; Schro"ttner, H.; Spießberger, C. Non-destructive detection of corrosion applied to steel and galvanized steel coated with organic paints by the pulse phase thermography. Materials and Corrosion 2012, 63, 195-199.

24. Fuhr, P. L.; Hutson, D. R. Corrosion detection in reinforced concrete roadways and bridges via embedded fiber optic sensors. Smart Mater. Struct. 1998, 7, 217-228.

25. Kendig, M. W.; Buchheit, R. G. Corrosion inhibition of aluminum and aluminum alloys by soluble chromates, chromate coatings, and chromate-free coatings. Corrosion 2003, 59, 379-400.

26. Briggs, M. S. J.; Fossey, J. S.; Richards, C. J.; Scott, B.; Whatelet, J. Towards novel biolabels: synthesis of a tagged highly fluorescent Schiff-base aluminum complex. Tet. Lett. 2002, 43, 5169-5171. 


\section{Appendix A: Epoxy Formulation}

Epon Starting Formulation 1003

\begin{tabular}{|c|c|}
\hline PART A & Weight $(\mathbf{g})$ \\
\hline Epon Resin 1001-CX-75 & 0.532 \\
\hline Beetle 216-7 & 0.345 \\
\hline PGME & 0.0672 \\
\hline MIBK & 0.0418 \\
\hline & \\
\hline PART B & 0.257 \\
\hline EPIKURE Curing Agent & 0.0675 \\
\hline PGME & $\mathbf{1 . 0 0}$ \\
\hline
\end{tabular}

